\title{
NONSELFADJOINT CROSSED PRODUCTS (INVARIANT SUBSPACES AND MAXIMALITY)
}

\author{
BY \\ MICHAEL MCASEY, PAUL S. MUHLY ${ }^{1}$ AND KICHI-SUKE SAITO ${ }^{2}$
}

\begin{abstract}
Let $\mathfrak{L}$ be the von Neumann algebra crossed product determined by a finite von Neumann algebra $M$ and a trace preserving automorphism. In this paper we investigate the invariant subspace structure of the subalgebra $\mathfrak{L}_{+}$of $\mathfrak{L}$ consisting of those operators whose spectrum with respect to the dual automorphism group on $\mathfrak{L}$ is nonnegative, and we determine conditions under which $\mathfrak{L}_{+}$is maximal among the $\sigma$-weakly closed subalgebras of $\mathfrak{R}$. Our main result asserts that the following statements are equivalent: (1) $M$ is a factor; (2) $\mathfrak{L}_{+}$is a maximal $\sigma$-weakly closed subalgebra of $\mathfrak{L}$; and (3) a version of the Beurling, Lax, Halmos theorem is valid for $\mathfrak{L}_{+}$. In addition, we prove that if $\mathfrak{A}$ is a subdiagonal algebra in a von Neumann algebra $\mathfrak{B}$ and if a form of the Beurling, Lax, Halmos theorem holds for $\mathfrak{A}$, then $\mathfrak{B}$ is isomorphic to a crossed product of the form $\mathfrak{R}$ and $\mathfrak{A}$ is isomorphic to $\mathfrak{R}_{+}$.
\end{abstract}

Introduction. Crossed products were introduced into operator theory by Murray and von Neumann in their first paper [14]. The algebras which they constructed as crossed products are now most commonly called group measure algebras. Subsequently, their construction was abstracted, generalized, and analyzed by numerous authors and it is fair to say that at present crossed products are ubiquitous in the theory of operator algebras. Indeed, Feldman and Moore [8] have recently shown that it is very likely that every von Neumann algebra can be realized as a crossed product-perhaps of a complicated nature. In this paper we consider von Neumann algebras which are constructed as very simple crossed products and focus our attention on certain nonselfadjoint subalgebras contained in them. Roughly speaking, the subalgebras we study stand in the same relation to the crossed products as the Hardy space $H^{\infty}(\mathbf{T})$, the space of boundary values of bounded analytic functions on the unit disc, stands in relation to the Lebesgue space $L^{\infty}(\mathbf{T})$.

Received by the editors November 11, 1977.

AMS (MOS) subject classifications (1970). Primary 46L15, 46L10, 46K05; Secondary 47C15.

Key words and phrases. Crossed products, von Neumann algebras, subdiagonal algebras, maximality questions.

${ }^{1}$ The second author was supported in part by a grant from the National Science Foundation.

${ }^{2}$ The third author would like to thank the Department of Mathematics of The University of Iowa for its support and hospitality during the course of this investigation. 
More specifically, suppose $M$ is a von Neumann algebra acting on a Hilbert space $^{3} \mathcal{H}$ and that $u$ is a unitary operator on $\mathcal{H}$ such that $u M u^{*}=M$. (Note that $u$ need not belong to $M$.) Form the Hilbert space $\mathbf{L}^{2}=l^{2}(\mathbf{Z}) \otimes \mathcal{H}$ and consider the operators $L_{x}, x \in M$, and $L_{\delta}$ defined on $\mathbf{L}^{2}$ by the formulae

$$
L_{x}=I \otimes x \quad \text { and } L_{\delta}=S \otimes u
$$

where $S$ is the usual bilateral shift on $l^{2}(\mathbf{Z})$. Then the von Neumann algebra crossed product determined by $M$ and (the automorphism implemented by) $u$ is defined to be the von Neumann algebra $\mathfrak{L}$ on $\mathbf{L}^{2}$ generated by $\left\{L_{x} \mid x \in M\right\}$ and $L_{\delta}$ while the subalgebra which we investigate and call a nonselfadjoint crossed product is the ultraweakly closed (or, as we shall write, the $\sigma$-weakly closed) algebra $\mathfrak{L}_{+}$generated by $\left\{L_{x} \mid x \in M\right\}$ and the positive powers of $L_{\delta}$. Observe that if $\mathcal{H}$ has dimension one so that $u$ is trivial, then $\mathfrak{L}$ is isomorphic to $L^{\infty}(\mathbf{T})$ and $\mathfrak{R}_{+}$is isomorphic to $H^{\infty}(\mathbf{T})$.

There are a variety of ways to define $\mathfrak{L}$ and $\mathfrak{L}_{+}$but up to isomorphism, these algebras depend only upon the isomorphism class of $M$ and on the automorphism implemented by $u$ [24]. Consequently for technical reasons we have decided to assume that $M$ is in standard form and to construct $\mathfrak{R}$ as the left von Neumann algebra associated with a certain Hilbert algebra; this explains the notation. In addition, we assume that $M$ is finite and that the automorphism implemented by $u$ preserves a faithful, normal, finite trace. Under these assumptions $\mathfrak{L}$ is finite and $\mathfrak{L}_{+}$is an example of what Arveson [2] calls a finite, maximal, subdiagonal algebra. Our objective in this paper is to investigate the lattice of subspaces invariant under $\mathfrak{L}_{+}, \operatorname{Lat}\left(\mathfrak{L}_{+}\right)$, and to determine when $\mathfrak{L}_{+}$is maximal among all the $\sigma$-weakly closed subalgebras of $\mathfrak{R}$. It turns out that invariant subspaces and questions of maximality are intimately related.

In [11], Loebl and Muhly presented an abstract and very general framework in which to investigate $\operatorname{Lat}\left(\mathfrak{L}_{+}\right)$. Although very useful for some purposes, the parameters presented there are quite cumbersome and really do not provide one with much insight into the structure of $\operatorname{Lat}\left(\mathfrak{L}_{+}\right)$. In this paper we abandon the approach in [11] and take as our starting point the observations that $L_{\delta}$ is a bilateral shift, an operator whose invariant subspace structure is well understood, and that the invariant subspaces of $\mathfrak{L}_{+}$are found among those of $L_{\delta}$. It turns out that when $M$ is a factor, then every subspace $\Re$ in Lat $\left(\mathfrak{L}_{+}\right)$which contains no subspace reducing $\mathfrak{L}_{+}$is of the form $\mathfrak{N}=R_{v} \mathbf{H}^{2}$ where $R_{v}$ is a partial isometry in the commutant, $\Re$, of $\mathfrak{R}$ and where $\mathbf{H}^{2}$ is the subspace $l^{2}\left(\mathbf{Z}_{+}\right) \otimes \mathscr{H}$ of $\mathbf{L}^{2}$. Thus when $M$ is a factor, a perfect analogue of the Beurling, Lax, Halmos theorem (hereafter abbreviated the BLH theorem)

\footnotetext{
${ }^{3}$ To avoid pathology all of our Hilbert spaces will be assumed to be separable and, of course, complex.
} 
is valid. This, by itself, is not altogether surprising nor is it difficult to prove. However, it develops that a conditioned converse is true. That is, if every $\mathfrak{R}$ in Lat $\left(\mathfrak{L}_{+}\right)$of a particular kind may be expressed as $\mathfrak{R}=R_{v} \mathbf{H}^{2}$ where $R_{v}$ is a unitary in $\Re$, then necessarily $M$ is a factor. Still more is true. We show that if $\mathfrak{A}$ is a finite, maximal, subdiagonal algebra in the sense of Arveson and if a form of the BLH theorem is valid for $\mathfrak{A}$, then the von Neumann algebra generated by $\mathfrak{A}$ is isomorphic to a crossed product of the form $\mathfrak{L}$ with $\mathfrak{A}$ identified with $\mathfrak{R}_{+}$. Thus, in a sense, the most general context in which one can expect the BLH theorem to hold is that of a nonselfadjoint crossed product where the coefficient algebra is a factor.

While a subdiagonal algebra $\mathfrak{A}$ in a von Neumann algebra $\mathfrak{B}$ may be maximal among the subdiagonal algebras in $\mathfrak{B}$, it need not be maximal among all the $\sigma$-weakly closed subalgebras of $\mathfrak{B}$. Indeed, most of the subdiagonal algebras studied by Arveson in [2] as well as most of those studied by Loebl and Muhly in [11] are all maximal as subdiagonal algebras but are not maximal among the $\sigma$-weakly closed subalgebras. Initially we could only identify a very small class of subdiagonal algebras which are maximal among the $\sigma$-weakly closed subalgebras of the von Neumann algebras they generate and, therefore, it became interesting to determine whether this class is exhaustive or are there more. There are more, and many can be found among nonselfadjoint crossed products. Specifically, we prove that $\mathfrak{L}_{+}$is a maximal $\sigma$-weakly closed subalgebra of $\mathfrak{L}$ if and only if $M$ is a factor. Thus maximality is tied to the validity of the BLH theorem. This may not be surprising in view of the fact that on the disc, the maximality of $H^{\infty}(\mathrm{T})$ as a weak-* closed subalgebra of $L^{\infty}(T)$ is a trivial consequence of Beurling's theorem, but in the present study, the matter is considerably more complicated.

Questions of maximality are more than idle curiosities, they reflect on fundamental structural properties of the algebras considered. In the theory of function algebras, where such questions are regarded as questions in abstract approximation theory, this has been known for some time. In operator theory, on the other hand, maximality plays a somewhat different role. Roughly speaking, an algebra $\mathfrak{A}$ is maximal in the von Neumann algebra $\mathfrak{B}$ it generates if and only if it is completely determined by its reducing subspaces-the subspaces which determine $\mathfrak{B}$-and any one of its nonreducing, invariant subspaces. This phenomenon is particularly important in the present investigation where we shall encounter it when we prove that a form of the BLH theorem is valid for $\mathfrak{L}_{+}$if and only if $M$ is a factor and when we prove that if a form of the BLH theorem is valid for a subdiagonal algebra, then it must be a nonselfadjoint crossed product.

The first two sections are concerned with preliminaries and somewhat technical material. $\S 1$ is devoted to a discussion of subdiagonal algebras in 
general and to a result which on the disc is an easy consequence of Szegö's theorem. Since, however, Szegö's theorem is not known to be valid in the context of subdiagonal algebras, a somewhat artful extension of an argument of Arveson is presented. The second section is concerned with crossed products. Here we describe formally the algebras which we are investigating and collect a number of known results for later use. We also prove a result which asserts that if $M$ is a factor but $\mathfrak{L}$ is not, a definite possibility, then the center of $\mathfrak{L}, Z(\mathfrak{Z})$, is normally ${ }^{*}$-isomorphic to $L^{\infty}(\mathbf{T})$ in such a way that $3(\mathfrak{R}) \cap \mathfrak{L}_{+}$is carried onto $H^{\infty}(\mathrm{T})$.

The main point of $\S 3$ is our version of the BLH theorem which is valid when $M$ is a factor. We consider also invariant subspaces contained in the other abstract Lebesgue spaces associated with $\mathfrak{L}$ in noncommutative integration theory. The point of this, ultimately, is to describe the $\sigma$-weakly closed ideals in $\mathfrak{R}_{+}$. In $\S 4$, we show the equivalence of the assertions that $M$ is a factor, a version of the BLH theorem is valid, and $\mathfrak{L}_{+}$is maximal among the $\sigma$-weakly closed subalgebras of $\mathfrak{R}$. Some consequences of our invariant subspace theorems are developed in $\$ 5$. Finally, in $\$ 6$, we prove that if a version of the BLH theorem is valid in a subdiagonal algebra then it must be a nonselfadjoint crossed product.

1. Preliminaries: subdiagonal algebras. In this paper we shall, for the most part, consider only finite von Neumann algebras. Moreover, they will almost always be in standard form. In order to emphasize the similarities between the noncommutative theory we are investigating and the classical function theory on the disc, we introduce the following notation. Some of it may seem pedantic, but we have found it useful to help separate the variety of roles played by the operators being considered. The reader is certainly aware of the fact that functions on the disc frequently play different roles, but usually it is not difficult to decide from context the interpretation intended. However, in the theory we are developing the situation becomes considerably more complicated primarily of course because the operators considered do not generally commute.

All traces without exception will be assumed to be finite, faithful, normal and normalized. If $M$ is a von Neumann algebra and if $\phi$ is a trace on $M$, then we shall denote the noncommutative Lebesgue spaces associated with $\boldsymbol{M}$ and $\phi$ by $L^{p}(M, \phi)$ or simply $L^{p}$ (see [6], [15], or [22]). As is customary, $M$ will be identified with $L^{\infty}$ while the ultraweak or $\sigma$-weak topology on $M$ will be identified with the weak-* topology on $L^{\infty}$ regarded as the dual of $L^{1}$ (cf. [7, p. 107]). The closure of a subset $\subseteq$ of $L^{p}$ in the $L^{p}$-norm, $1 \leqslant p<\infty$, will be denoted by $[\subseteq]_{p} ;[\subseteq]_{\infty}$ will denote the closure of $\subseteq$ in the weak-* topology on $L^{\infty}$.

If $x$ is in $M$, we shall write $L_{x}$ (resp. $R_{x}$ ) for the operator defined (on any 
$L^{p}$-space) by the equation $L_{x} f=x f$ (resp. $R_{x} f=f x$ ), $f \in L^{p}$, and we let $\mathfrak{L}$ (resp. $\mathfrak{R}$ ) denote the algebra of all such operators. One may regard $M$ as a finite, achieved Hilbert algebra whose completion is $L^{2}$, and when this is done, $\mathfrak{L}$ and $\Re$ are the left and right von Neumann algebras of $M$ and the map $x \rightarrow L_{x}$ (resp. $x \rightarrow R_{x}$ ) is a normal, *-isomorphism (resp. *-anti-isomorphism) of $M$ onto $\mathfrak{R}$ (resp. $\Re$ ). As a consequence, $\mathfrak{L}^{\prime}=\mathfrak{R}$ and the identity $I$ of $M$ is a cyclic and separating vector for $\mathfrak{L}$ and $\mathfrak{R}$ (cf. [7, Chapitre $I, \S 5]$ ). Sometimes elements $f$ in $L^{p}$ will be regarded as (possibly) unbounded operators affiliated with $\mathfrak{L}$ or $\mathfrak{R}$. Finally, the canonical conjugate-linear, isometric involution on $L^{2}$ which extends the map $x \rightarrow x^{*}$ on $M$ will be denoted by $J$.

In [2], Arveson introduced the concept of a subdiagonal algebra for the purpose of providing a unified approach to the analysis of a variety of rather broad classes of nonselfadjoint operator algebras. These algebras are best thought of as a noncommutative generalization of the weak-* Dirichlet algebras of Srinivasan and Wang [23]. The most tractable are the finite maximal ones, and these are the only kind considered here.

Definition. Let $\mathfrak{B}$ be a von Neumann algebra with trace $\phi$, let $\mathfrak{A}$ be a $\sigma$-weakly closed subalgebra of $\mathfrak{B}$, and let $\Phi$ be a faithful, normal expectation from $\mathfrak{B}$ onto $\mathfrak{D}=\mathfrak{A} \cap \mathfrak{U}^{*}\left(\mathfrak{H}^{*}=\left\{x^{*} \mid x \in \mathfrak{A}\right\}\right)$. Then $\mathfrak{A}$ is called a finite, maximal, subdiagonal algebra in $\mathscr{B}$ with respect to $\Phi$ and $\phi$ in case the following conditions are satisfied:

(1) $\mathfrak{A}+\mathfrak{A}^{*}$ is $\sigma$-weakly dense in $\mathfrak{B}$;

(2) $\Phi(x y)=\Phi(x) \Phi(y)$, for all $x$ and $y$ in $\mathfrak{A}$;

(3) $\mathfrak{A}$ is maximal among those subalgebras of $\mathfrak{B}$ satisfying (1) and (2); and

(4) $\phi \circ \Phi=\phi$.

Concerning this definition we note that any subalgebra $\mathfrak{A}$ of $\mathfrak{B}$ satisfying (1) and (2) can be imbedded in a unique algebra $\mathfrak{A}_{m}$ which is maximal among all algebras satisfying (1) and (2). In fact, $\mathfrak{A}_{m}=\{x \in \mathfrak{B} \mid \Phi(t x a)=\Phi(a x t)=$ $0, a \in \mathfrak{A}$ and $t \in \mathfrak{A}$ such that $\Phi(t)=0\}$ [2, Theorem 2.2.1]. In the presence of condition (4), $\mathfrak{U}_{m}=\{x \in \mathfrak{B} \mid \Phi(x t)=0, t \in \mathfrak{A}, \Phi(t)=0\}$ [2, Corollary 2.2.4]. We wish to emphasize once more that while a subdiagonal algebra in a von Neumann algebra $\mathfrak{B}$ may be maximal as a subdiagonal algebra in $\mathfrak{B}$, it need not be maximal among the $\sigma$-weakly closed subalgebras of $\mathfrak{B}$.

For $1 \leqslant p<\infty$, the closure of $\mathfrak{A}$ in $L^{p}(\mathfrak{B}, \phi)$ is denoted by $H^{p}(\mathfrak{B}, \phi)$ or $H^{p}$ and the closure of $\operatorname{ker}(\Phi \mid \mathfrak{A})$ is denoted by $H_{0}^{p}$. We identify $\mathfrak{A}$ with $H^{\infty}$ in the identification of $\mathfrak{B}$ with $L^{\infty}$ and $\operatorname{ker}(\Phi \mid \mathfrak{A})$ is identified with $H_{0}^{\infty}$. Also, we write $\mathfrak{L}_{+}$and $\mathfrak{R}_{+}$for $\mathfrak{L}(\mathfrak{U})$ and $\mathfrak{R}(\mathfrak{U})$.

We present now some technical, but useful, facts about subdiagonal algebras in general which will be called upon several times in the sequel. For these, we fix for the remainder of this section a finite von Neumann algebra $\mathfrak{B}$ with trace $\phi$, an expectation $\Phi$ on $\mathfrak{B}$, and a finite, maximal, subdiagonal algebra $\mathfrak{A}$ (with respect to $\Phi$ and $\phi$ ) in $\mathfrak{B}$. 
Proposition 1.1. The expectation $\Phi$ may be extended to $L^{2}$ and the extension is the orthogonal projection of $L^{2}$ onto $[\mathfrak{D}]_{2}$. In addition, $L^{2}$ may be decomposed into the orthogonal direct sum $L^{2}=H^{2} \oplus J H_{0}^{2}=H_{0}^{2} \oplus[\mathfrak{D}]_{2} \oplus J H_{0}^{2}$.

Proof. The first assertion is clear since $\phi$ preserves $\Phi$ and so by definition, $H_{0}^{2}=[\mathfrak{D}]_{2} \oplus H_{0}^{2}$. The fact that $H^{2} \oplus J H_{0}^{2}=L^{2}$ is proved on p. 583 in [2] in the proof of Theorem 2.2.1.

A frequently used corollary to Szegö's theorem is the assertion that if $k$ is a square-integrable function on the circle such that $\log |k|$ is also integrable, then there is an outer function $f$ in $H^{2}$ such that $|f|=|k|$. Although Szegö's theorem and this corollary are valid in the context of weak-* Dirichlet algebras [23], it is not known at this time if they have extensions to the context of subdiagonal algebras. We have found, however, that the next proposition is a very serviceable substitute for the corollary; it refines Theorem 4.2.1 of [2]. The proof follows Arveson's arguments rather closely, but at a number of points certain adjustments which are not altogether obvious are required.

PROPOSITION 1.2. If $k$ is in $L^{\infty}(=\mathfrak{B})$ with (possibly unbounded) inverse lying in $L^{2}$, then there are unitary operators $u_{1}$ and $u_{2}$ in $L^{\infty}$ and operators $a_{1}$ and $a_{2}$ in $H^{\infty}(=\mathfrak{U})$ such that $k=u_{1} a_{1}=a_{2} u_{2}$.

Proof. We prove that $k=u_{1} a_{1}$; the other representation is verified in a similar fashion. First note that $k$ does not belong to $\left[k H_{0}^{\infty}\right]_{2}$ because if it did, then $k$ would be the limit, $\lim k a_{n}$, for a suitable sequence $\left\{a_{n}\right\}_{n=0}^{\infty}$ in $H_{0}^{\infty}$. But then, since $\phi\left(a_{n}\right)=\phi\left(\Phi\left(a_{n}\right)\right)=0$,

$$
\begin{aligned}
1=\phi(I) & =\lim \phi\left(I-a_{n}\right)=\lim \phi\left(k^{-1}\left(k-k a_{n}\right)\right) \\
& =\lim \left(k-k a_{n},\left(k^{-1}\right)^{*}\right)=0,
\end{aligned}
$$

a contradiction. Write $k=\zeta+\eta$ where $\eta$ is the projection of $k$ on $\left[k H_{0}^{\infty}\right]_{2}$. Then $\zeta$ is orthogonal to $\left[\zeta H_{0}^{\infty}\right]_{2}$; i.e., $\zeta$ is right wandering in the sense of Arveson. Indeed, since $\eta$ lies in $\left[k H_{0}^{\infty}\right]_{2}$, a subspace of $L^{2}$ invariant under $\Re_{+}$, we find that $\left[\zeta H_{0}^{\infty}\right]_{2}=\left[(k-\eta) H_{0}^{\infty}\right]_{2} \subseteq\left[k H_{0}^{\infty}+\eta H_{0}^{\infty}\right]_{2}=\left[k H_{0}^{\infty}\right]_{2}$; and since $\zeta$ is orthogonal to $\left[k H_{0}^{\infty}\right]_{2}$, it is also orthogonal to $\left[\zeta H_{0}^{\infty}\right]_{2}$. By Lemma 4.2.2 of [2], there is a partial isometry $u$ in $L^{\infty}$ such that $u \zeta$ lies in [D] $]_{2}$, say $u \zeta=\zeta_{0} ; L_{u}^{*} L_{u}$ is the projection onto $\left[\zeta L^{\infty}\right]_{2} ;$ and $L_{u} L_{u}^{*}$ is the projection onto $\left[\zeta_{0} L^{\infty}\right]_{2}$. We assert that $u$ is unitary. Since $L^{\infty}$ is a finite von Neumann algebra, it suffices to prove that $\left[\zeta L^{\infty}\right]_{2}=L^{2}$. For this, Arveson $[2$, p. 603] argues that it suffices to prove that $\zeta$ is a separating vector for $\Re$. If $R_{x} \zeta=\zeta x=0$ for some $x$ in $L^{\infty}, x \neq 0$, then another argument on p. 603 of [2] shows that $\zeta h=0$ for a nonzero $h$ in $\mathfrak{D}$. We may of course assume that 
$h \geqslant 0$. Now observe that $k h=\zeta h+\eta h=\eta h$ lies in $\left[k H_{0}^{\infty}\right]_{2}$. So, if $\left\{a_{n}\right\}_{n=0}^{\infty}$ is a sequence in $H_{0}^{\infty}$ such that $\lim \left\|k h-k a_{n}\right\|_{2}=0$, then since $\phi\left(a_{n}\right)=\phi\left(\Phi\left(a_{n}\right)\right)$ $=0$, we find that

$$
\begin{aligned}
\phi(h) & =\lim \phi\left(h-a_{n}\right)=\lim \left(k^{-1}\left(k h-k a_{n}\right), I\right) \\
& =\lim \left(\left(k h-k a_{n}\right),\left(k^{-1}\right)^{*}\right)=0 .
\end{aligned}
$$

But $h \geqslant 0$ and $\phi$ is faithful, so $h=0-$ a contradiction. To complete the proof, we need only show that $u k$ lies in $H^{\infty}$. By Proposition 1.1 and Corollary 2.2.4 of [2], it suffices to prove that for each $y$ in $H_{0}^{\infty}, u k y$ is orthogonal to [D] $]_{2}$. Since, as we just showed, $\zeta$ is a cyclic and separating vector for $\Re$, and since $\zeta_{0}=u \zeta$ with $u$ unitary, $\zeta_{0}$ is also a cyclic and separating vector for $\Re$. But $\zeta_{0}$ belongs to $[\mathfrak{D}]_{2}$ and $\Re(\mathfrak{D}) \mid[\mathfrak{D}]_{2}$ has $I$ as a cyclic vector, therefore by Proposition 4 on p. 221 of [7] and the fact that $\Re(D)$ is finite, $\zeta_{0}$ is a cyclic vector for $\Re(\mathfrak{D}) \mid[\mathfrak{D}]_{2}$; i.e., $\left[\zeta_{0} \mathfrak{D}\right]_{2}=[\mathfrak{D}]_{2}$. Thus we need to see if $u k y$ is orthogonal to $\zeta_{0} d$ for all $y$ in $H_{0}^{\infty}$ and $d$ in $\mathfrak{D}$. But this is easy:

$$
\begin{aligned}
\left(u k y, \zeta_{0} d\right) & =\left(k y, u^{*} \zeta_{0} d\right)=(k y, \zeta d) \\
& =\left(k y, R_{d} \zeta\right)=\left(R_{d}^{*} k y, \zeta\right)=\left(k y d^{*}, \zeta\right)=0
\end{aligned}
$$

because $y d^{*} \in H_{0}^{\infty}, k y d^{*} \in\left[k H_{0}^{\infty}\right]_{2}$, and $\zeta$ is orthogonal to $\left[k H_{0}^{\infty}\right]_{2}$ by definition. With this, the proof is complete.

COROLlaRY 1.3. If $\mathscr{N}$ is a closed subspace of $L^{p}, 1 \leqslant p<\infty$, which is invariant either under $\mathfrak{L}_{+}$or $\Re_{+}$, then $\mathfrak{T}_{\cap} \cap L^{\infty}$ contains elements different from zero.

Proof. As will be seen in the course of the proof, we may assume without loss of generality that $p=1$. Also, we shall assume that $\Re_{+} \mathfrak{N} \subseteq \mathfrak{K}$. Let $\xi$ be a nonzero element in $\Re$ and consider its polar decomposition: $\xi=v|\xi|=$ $v|\xi|^{1 / 2}|\xi|^{1 / 2}$. Let $f$ be the function on $[0, \infty)$ defined by the formula $f(x)=1$, $0 \leqslant x \leqslant 1, f(x)=1 / x, x>1$, and define $k$ to be $f\left(|\xi|^{1 / 2}\right)$ through the functional calculus. Then $k$ is in $L^{\infty}, k^{-1}$ is in $L^{2}$, and $|\xi|^{1 / 2} k$ is in $L^{\infty}$. By the preceding proposition we may choose a unitary $u$ in $L^{\infty}$ and an $a$ in $H^{\infty}$ such that $a u=k$. Then $\xi a=v|\xi|^{1 / 2}|\xi|^{1 / 2} k u^{*}$, which shows clearly that $\xi a$ is a nonzero element in $L^{2}$, and $\xi a$ lies in $\mathfrak{N}$ because $\mathfrak{N}$ is $\Re_{+}$invariant. Thus $\Re \cap L^{2}$ contains nonzero elements. But the argument may now be reapplied to produce a $b$ in $H^{\infty}$ such that $\xi a b$ is a nonzero element in $\Re \cap L^{\infty}$.

REMARK 1.4. It is attractive to conjecture that $\mathfrak{N}$ is the closure of the space of bounded elements it contains. This is of course true in the weak-* Dirichlet algebra setting (cf. $[10, \S 1.6]$ ) but the proofs that we know seem to use Szegö's theorem in a fairly sophisticated way. We are able to prove the conjecture in 
the case of certain crossed products only after considerable preparation (see Corollary 3.8).

COROLLARY 1.5. If $\mathbb{E}$ is a proper $\sigma$-weakly closed subalgebra of $\mathfrak{B}$ containing $\mathfrak{U}$, then $[\mathbb{\complement}]_{2} \neq L^{2}$.

Proof. Let $\mathfrak{N}$ be the annihilator of $\mathbb{E}$ in $L^{1}$. By hypothesis, $\mathscr{T} \neq\{0\}$. Since $\mathfrak{A} \subseteq \mathfrak{E}, \mathfrak{N}$ is $\mathfrak{R}_{+}$invariant. By the preceding corollary $\mathfrak{N}$ contains nonzero bounded elements and these, or more accurately their adjoints, are orthogonal to $[\Subset]_{2}$ in $L^{2}$.

2. Crossed products. As noted in the Introduction, there are a variety of ways to define crossed products. The most congenial way from our point of view here is to define first a Hilbert algebra and then to take an associated von Neumann algebra as the crossed product. To this end we fix for the remainder of this paper a finite von Neumann algebra $M$ and a trace $\phi$ on $M$. We assume $M$ is in standard form and identify it when convenient with either the von Neumann algebra of left multiplications on $L^{2}=L^{2}(M, \phi)$, the von Neumann algebra of right multiplications, or with $L^{\infty}$ as we did in the preceding section. Also, we fix once and for all a normal, ${ }^{*}$-automorphism $\alpha$ of $M$ which preserves $\phi$; i.e., $\phi \circ \alpha=\phi$. The following proposition is easily proved and so the proof will be omitted.

Proposition 2.1. Let $\mathbf{L}_{0}^{2}=\{f: \mathbf{Z} \rightarrow M \mid f(n)=0$ for all but finitely many $n\}$. Then with respect to pointwise addition and scalar multiplication and the operations defined by equations (1)-(3), $\mathbf{L}_{0}^{2}$ is a Hilbert algebra with identity $\psi$ defined by $\psi(0)=I_{M}$, and $\psi(n)=0, n \neq 0$.

(1) $(f * g)(n)=\sum_{k \in \mathbf{Z}} f(k) \alpha^{k}(g(n-k))$,

(2) $\left(f^{*}\right)(n)=\left[\alpha^{n}(f(-n))\right]^{*}$,

(3) $(f, g)=\sum_{k \in \mathbf{Z}}(f(k), g(k))_{L^{2}(m, \phi)}$.

It is helpful to think of $\mathbf{L}_{0}^{2}$ as the space of all "twisted", $M$-valued trigonometric polynomials. That is, if the powers of $\alpha$ were absent in 2.1 , then $\mathbf{L}_{0}^{2}$ would indeed consist of the set of all coefficients of $M$-valued trigonometric polynomials and 2.1 would simply be ordinary convolution. Observe, too, that the Hilbert space completion $\mathbf{L}^{2}$ of $\mathbf{L}_{0}^{2}$ is precisely

$$
\left\{f: \mathbf{Z} \rightarrow L^{2}(M, \phi) \mid \sum_{n \in \mathbf{Z}}\|f(n)\|_{L^{2}}^{2}<\infty\right\}
$$

and may be identified with $l^{2}(\mathbf{Z}) \otimes L^{2}(M, \phi)$.

For $f$ in $\mathbf{L}_{0}^{2}$, we define operators $L_{f}$ and $R_{f}$ on $\mathbf{L}^{2}$ by the formulas $L_{f} g=f * g$ and $R_{f} g=g * f, g \in \mathbf{L}^{2}$. Note that since the sums defining $L_{f}$ and $R_{f}$ are finite and since $f(n) \in M$ for all $n$, both $L_{f}$ and $R_{f}$ are well defined, bounded operators on $\mathbf{L}^{2}$. As is customary, we set $\mathfrak{L}=\left\{L_{f} \mid f \in \mathbf{L}_{0}^{2}\right\}^{\prime \prime}$ 
and $\Re=\left\{R_{f} \mid f \in \mathbf{L}_{0}^{2}\right\}^{\prime \prime}$ by definition. Also, we define $\mathbf{L}^{\infty}$ to be the achieved Hilbert algebra of all bounded elements in $\mathbf{L}^{2}$. That is, $\mathbf{L}^{\infty}$ consists of those $f$ in $\mathbf{L}^{2}$ such that the map $g \rightarrow f * g, g \in \mathbf{L}_{0}^{2}$, extends to a bounded operator on all of $\mathbf{L}^{2}$. For such an $f$, we write $L_{f}$ and $R_{f}$ for the operators it determines. It is of course a basic fact from Hilbert algebra theory (cf. [7, Chapitre I, §5]) that since $\mathbf{L}_{0}^{2}$ and hence $\mathbf{L}^{\infty}$ has an identity, the map $f \rightarrow L_{f}$ is a *-isomorphism from $\mathbf{L}^{\infty}$ onto $\mathfrak{L}$ while the map $f \rightarrow R_{f}$ is a *-anti-isomorphism mapping $\mathbf{L}^{\infty}$ onto $\mathfrak{R}$. Moreover, $\mathfrak{L}$ and $\mathfrak{R}$ are commutants of one another. Since $\alpha$ preserves $\phi$ on $M, \alpha$ extends to a unitary operator on $L^{2}(M, \phi)$. Consequently, the canonical antiunitary involution $J$ on $\mathbf{L}^{2}$, extending the ${ }^{*}$ operation on $\mathbf{L}_{0}^{2}$, is given by the formula (2). Also, since $\mathbf{L}_{0}^{2}$ has an identity $\psi, \mathfrak{R}$ is a finite von Neumann algebra and the functional $\tau$ on $\mathfrak{L}$ defined by $\tau\left(L_{f}\right)=$ $(f, \psi)=\phi(f(0)), f \in \mathbf{L}^{\infty}$, is readily seen to be a finite, faithful, normal and normalized trace (cf. [7, p. 85]). We abuse notation a little and write $\tau\left(L_{f}\right)=\tau\left(R_{f}\right)=\tau(f)$.

At this point it should be remarked that the notation we have introduced is quite consistent with that presented in $\S 1$. That is, $\mathfrak{L}$ is a finite von Neumann algebra in standard form, $\mathbf{L}^{2}=L^{2}(\mathfrak{\Omega}, \tau), \mathbf{L}^{\infty}=L^{\infty}(\mathfrak{R}, \tau)$, etc. We call $\mathbf{L}^{\infty}$ the selfadjoint or von Neumann algebra crossed product determined by $M, \phi$, and $\alpha$ and refer to $\mathfrak{R}$ and $\Re$ as the left and right regular representations of it.

The original algebra $M$ is identified with the subalgebra $\{x \psi \mid x \in M\}$ of $\mathbf{L}^{\infty}$, and we abbreviate $L_{x \psi}$ and $R_{x \psi}$ by $L_{x}$ and $R_{x}$. It is instructive to note that $L_{x}$ is the infinite ampliation $\left(L_{x} f\right)(n)=x f(n)$ while $\left(R_{x} f\right)(n)=f(n) \alpha^{n}(x)$, $f \in \mathbf{L}^{2}$. As before, we write $\mathfrak{L}(M)=\left\{L_{x} \mid x \in M\right\}$ and $\mathfrak{R}(M)=\left\{R_{x} \mid x \in M\right\}$. The function $\delta$, defined by the formula

$$
\delta(n)=\left\{\begin{array}{cc}
I_{M}, & n=1 \\
0, & n \neq 1
\end{array}\right.
$$

plays a very important role. For, as a straightforward calculation reveals, $\mathfrak{L}=\left\{\mathfrak{L}(\mathrm{M}), L_{\delta}\right\}^{\prime \prime}$ and $\Re=\left\{\Re(M), R_{\delta}\right\}^{\prime \prime}$. It is also instructive to note that when $\mathbf{L}^{2}$ is identified with $l^{2}(\mathbf{Z}) \otimes L^{2}(M, \phi)$, then $L_{\delta}$ becomes $S \otimes u$ and $R_{\delta}$ becomes $S \otimes I_{M}$ where $S$ is the bilateral shift on $L^{2}(Z)$ and $u$ is the unitary operator on $L^{2}(M, \phi)$ induced by $\alpha$. It is now clear, of course, that the algebras we are constructing are the same as those presented in the Introduction.

The automorphism group $\left\{\beta_{t}\right\}_{t \in \mathbf{R}}$ of $\mathfrak{L}$ dual to $\alpha$ in the sense of Takesaki [24] is implemented by the unitary representation of $\mathbf{R},\left\{W_{t}\right\}_{t \in \mathbf{R}}$, defined by the formula $\left(W_{t} f\right)(n)=e^{2 \pi i n t} f(n), f \in \mathbf{L}^{2}$; that is, $\beta_{t}\left(L_{f}\right)=W_{t} L_{f} W_{t}^{*}$, by definition. Similarly, we define $\beta_{t}\left(R_{f}\right)=W_{t} R_{f} W_{t}^{*}$. It is easy to see that $\beta_{t}\left(L_{f}\right)=$ $L_{W f}$ for all $f$ in $\mathbf{L}^{\infty}$ and similarly for $R_{f}$. Indeed, if $g$ is in $\mathbf{L}^{2}$ and $f$ is in $\mathbf{L}^{\infty}$, 
then

$$
\begin{aligned}
\left(\beta_{t}\left(L_{f}\right) g\right)(n) & =\left(W_{t} L_{f} W_{t}^{*} g\right)(n)=e^{2 \pi i n t}\left(L_{f} W_{t}^{*} g\right)(n) \\
& =e^{2 \pi i n t}\left(f * W_{t}^{*} g\right)(n)=e^{2 \pi i n t} \sum_{k \in \mathbf{Z}} f(k) \alpha^{k}\left(\left(W_{t}^{*} g\right)(n-k)\right) \\
& =e^{2 \pi i n t} \sum_{k \in \mathbf{Z}} f(k) e^{2 \pi i(k-n) t} \alpha^{k}(g(n-k)) \\
& =\sum_{k \in \mathbf{Z}} e^{2 \pi i k t} f(k) \alpha^{k}(g(n-k)) \\
& =\left(\left(W_{t} f\right) * g\right)(n)=\left(L_{W_{f}} g\right)(n) .
\end{aligned}
$$

On account of this, we write $W_{t}(f)=\beta_{t}(f)$ when $f$ is in $\mathbf{L}^{\infty}$.

It is elementary to check that the spectral resolution of $\left\{W_{t}\right\}_{t \in \mathbf{R}}$ is given by the formula

$$
W_{t}=\sum_{n=-\infty}^{\infty} e^{2 \pi i n t} E_{n}
$$

where $E_{n}$ is the projection on $\mathbf{L}^{2}$ defined by the formula

$$
\left(E_{n} f\right)(k)=\left\{\begin{array}{cc}
f(n), & k=n, \\
0, & k \neq n .
\end{array}\right.
$$

It is equally easy to check that the projection $E_{n}$ can be calculated as the (Bochner) integral

$$
E_{n}(f)=\int_{0}^{1} e^{-2 \pi i n t} W_{t}(f) d t .
$$

The restriction on $E_{n}$ to $\mathrm{L}^{\infty}$ will be denoted by $\varepsilon_{n}$ and we shall write $\varepsilon_{n}\left(L_{f}\right)=L_{\varepsilon_{n}(f)}$ and $\varepsilon_{n}\left(R_{f}\right)=R_{\varepsilon_{n}(f)}$ as well. Of course we may write

$$
\varepsilon_{n}=\int_{0}^{1} e^{-2 \pi i n t} \beta_{t} d t
$$

but where the integral converges in the $\sigma$-weak topology when applied to operators. We note that the $\varepsilon_{n}$ are ultraweakly continuous, linear maps, and in particular, the map $\varepsilon_{0}$ is a faithful, normal expectation, preserving $\tau$, from $\mathbf{L}^{\infty}$ onto the space of functions $f$ in $\mathbf{L}^{\infty}$ such that $f(n)=0$ when $n \neq 0$. The spectral subspaces of $\mathbf{L}^{\infty}, \mathfrak{L}$ and $\Re$ associated with $\left\{\beta_{t}\right\}_{t \in \mathbf{R}}$ as defined and analyzed in [2], [11], or [16], are related to the $\varepsilon_{n}$ 's by the prescription: If $S \subseteq \mathbf{Z}$, then the spectral subspace of $\mathbf{L}^{\infty}$ determined by $S$ and $\left\{\beta_{t}\right\}_{t \in \mathbf{R}}$, $\mathbf{L}_{\beta}^{\infty}(S)$, is $\left\{f \in \mathbf{L}^{\infty} \mid \varepsilon_{n}(f)=0, n \in \mathbf{Z} \backslash S\right\}$. All these assertions are easy to verify from the definitions but some verifications are tedious. In any event, they appear in various places in the literature and so we shall not pause to prove them here.

We define $\mathbf{H}^{2}=\left\{f \in \mathbf{L}^{2} \mid f(n)=0, n<0\right\}$, we define $\mathbf{H}^{\infty}$ to be $\mathbf{L}^{\infty} \cap \mathbf{H}^{2}$ are we refer to it as the nonselfadjoint crossed product determined by $M$ and $\alpha$. Alternatively, $\mathbf{H}^{\infty}=\mathbf{L}_{\beta}^{\infty}\left(\mathbf{Z}_{+}\right)$. Also, we set $\mathfrak{L}_{+}=\left\{L_{f} \mid f \in \mathbf{H}^{\infty}\right\}$ and $\Re_{+}=$ 
$\left\{R_{f} \mid f \in \mathbf{H}^{\infty}\right\}$. These objects are the principal structures studied in this paper and the following theorem summarizes their basic properties. For the proof, see $[11, \S 4.3]$ or $[20$, Theorem 2].

THEOREM 2.2. The space $\mathbf{H}^{\infty}$ is a finite, maximal subdiagonal algebra in $\mathbf{L}^{\infty}$ with respect to the expectation $\varepsilon_{0}$ and trace $\tau$. The diagonal of $\mathbf{H}^{\infty}$ consists of all those $f$ in $\mathbf{L}^{\infty}$ such that $f(n)=0, n \neq 0$. In addition, the map $f \rightarrow L_{f}$ (resp. $\left.f \rightarrow R_{f}\right), f \in \mathbf{H}^{\infty}$, is a $\sigma$-weakly continuous, isometric isomorphism (resp. antiisomorphism) of $\mathbf{H}^{\infty}$ onto $\mathfrak{L}_{+}\left(\right.$resp. $\left.\mathfrak{R}_{+}\right)$, mapping the diagonal of $\mathbf{H}^{\infty}$ onto $\mathfrak{L}(M)$ (resp. $\mathfrak{R}(M))$, and $\mathfrak{L}_{+}\left(\right.$resp. $\left.\mathfrak{R}_{+}\right)$is the $\sigma$-weakly closed algebra generated by $L_{\delta}$ and $\mathfrak{L}(M)$ (resp. $R_{\delta}$ and $\Re(M)$ ).

The von Neumann algebra $\mathbf{L}^{\infty}$ may or may not be a factor. This depends on the action of $\alpha$ on $M$. When $\mathbf{L}^{\infty}$ is not a factor but $M$ is, the next theorem identifies the intersection $3\left(\mathbf{L}^{\infty}\right) \cap \mathbf{H}^{\infty}$, where $3\left(\mathbf{L}^{\infty}\right)$ is the center of $\mathbf{L}^{\infty}$, with the classical Hardy space $H^{\infty}(\mathbf{T})$. We actually prove a slightly more general assertion.

THEOREM 2.3. Suppose that $\mathbf{L}^{\infty}$ is not a factor but that $3\left(\mathbf{L}^{\infty}\right) \cap M=\{\mathbf{C} I\}$. Then there is a normal ${ }^{*}$-isomorphism carrying $3\left(\mathbf{L}^{\infty}\right)$ onto $L^{\infty}(\mathbf{T})$ in such a way that $3\left(\mathbf{L}^{\infty}\right) \cap \mathbf{H}^{\infty}$ is carried onto $H^{\infty}(\mathbf{T})$.

Proof. Observe that the fixed point algebra of $\left\{\beta_{t}\right\}_{t \in \mathbf{R}}$ is $M$ where $M$ is regarded as the space of functions $f$ in $\mathbf{L}^{\infty}$ such that $f(n)=0$ when $n \neq 0$. Thus, since $3\left(\mathbf{L}^{\infty}\right) \cap M=\{\mathbf{C} I\}$, when $\left\{\beta_{t}\right\}_{t \in \mathbf{R}}$ is restricted to $3\left(\mathbf{L}^{\infty}\right)$, it acts ergodically. Since $\mathbf{L}^{2}$ is separable, $3\left(\mathbf{L}^{\infty}\right)$ is normally ${ }^{*}$-isomorphic to $L^{\infty}(X)$ for a suitable standard Borel space $X$. Using this isomorphism, $\left\{\beta_{t}\right\}_{t \in \mathbf{R}}$ may be identified with a group of *-automorphisms of $L^{\infty}(X)$ which, by a theorem of Mackey [12], is implemented by a measurable action of $\mathbf{R}$ on $X$. Thus we may write $\left[\beta_{t}(\varphi)\right](x)=\varphi(x+t), \varphi \in L^{\infty}(X)$, where $x+t$ denotes the translate of $x$ in $X$ by $t$ in $\mathbf{R}$. Since $\left\{\beta_{t}\right\}_{t \in \mathbf{R}}$ is ergodic and periodic, we may apply a result on p. 236 of [18] to conclude that except for a null set, $X$ consists of one periodic orbit. This we may identify with the circle group $\mathbf{T}$. When all the identifications we have made are composed, we find that the restriction of $\tau$ to $3\left(\mathbf{L}^{\infty}\right)$ is identified with Lebesgue measure, $3\left(\mathbf{L}^{\infty}\right)$ is identified with $L^{\infty}(\mathbf{T})$, $\left\{\beta_{t}\right\}_{t \in \mathbf{R}}$ is identified with the usual action of $\mathbf{R}$ on $\mathbf{T}$, and $3\left(\mathbf{L}^{\infty}\right) \cap \mathbf{H}^{\infty}$ is identified with all those functions in $L^{\infty}(\mathbf{T})$ whose Fourier coefficients of negative index vanish. This last space is, of course, $H^{\infty}(\mathbf{T})$.

We conclude this section with several examples which serve to illustrate the notions we have introduced.

EXAMPLE 2.4. Let $M$ be an arbitrary finite von Neumann algebra but suppose the automorphism $\alpha$ is inner, implemented, say, by a unitary $u$ in $M$. Then since $L_{u}^{*} L_{\delta}=R_{\delta}$, so that $R_{\delta}$ belongs to $\mathfrak{L}$, it is easy to check that $\mathbf{L}^{\infty}$ is 
isomorphic to $L^{\infty}(\mathbf{T}) \otimes M$ in such a way that $\mathbf{H}^{\infty}$ is carried to $H^{\infty}(\mathbf{T}) \otimes M$. Thus, if we specialize further and assume that $M$ is the algebra $\mathbf{M}_{n}$ of all complex $n \times n$ matrices, we find that the theory we are developing contains (most of) the vectorial function theory developed by Helson and Lowdenslager, Masani and Wiener, and others for use in prediction theory and related areas.

EXAMPLE 2.5. Let $\Omega=\left\{\omega_{0}, \omega_{1}, \ldots, \omega_{n-1}\right\}$ be a finite set, let $M=l^{\infty}(\Omega)$, and let $\alpha$ be implemented by the cyclic permutation $\omega_{0} \rightarrow \omega_{1} \rightarrow \cdots \rightarrow \omega_{n-1}$ $\rightarrow \omega_{0}$. Using basic principles, it is easy to show that $\mathbf{L}^{\infty}$ is isomorphic to $L_{\infty}(\mathbf{T}) \otimes \mathbf{M}_{n}$. But more is true. Identify $L^{\infty}(\mathbf{T}) \otimes \mathbf{M}_{n}\left(\right.$ resp. $\left.H^{\infty}(\mathbf{T}) \otimes \mathbf{M}_{n}\right)$ with the $n \times n$ matrices $\left[a_{i j}\right]$ such that $a_{i j} \in L^{\infty}(\mathbf{T})$ (resp. $a_{i j} \in H^{\infty}(\mathbf{T})$ ) and denote the characteristic function of the singleton $\left\{\omega_{k}\right\}$ in $\Omega$ by $x_{k}$. Then $\Psi$, defined on generators by the equations below, extends to be a ${ }^{*}$-isomorphism of $\mathfrak{L}$ onto $L^{\infty}(\mathbf{T}) \otimes \mathbf{M}_{n}$ carrying $\mathfrak{L}_{+}$onto the matrices $\left[a_{i j}\right]$ in $H^{\infty}(\mathbf{T}) \otimes \mathbf{M}_{n}$ such that $a_{i j}(0)=0$ if $a_{i j}$ is above the main diagonal.

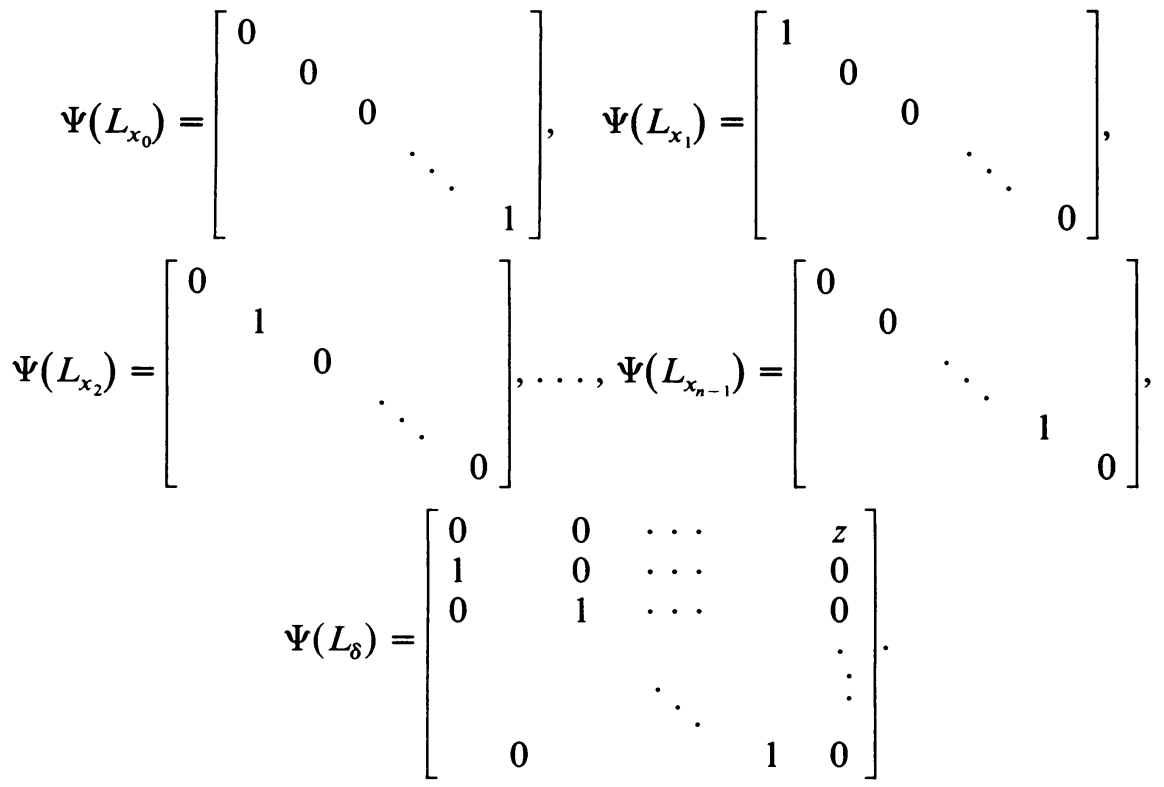

A detailed analysis of this example and the two to follow is carried out by the first author in [13].

EXAMPLE 2.6. This example does not quite fall within the scope of this investigation, but we feel that it is sufficiently close to the algebras analyzed here that it warrants inclusion, particularly because it is so simple. Let $M=l^{\infty}(\mathbf{Z})$ and let $\alpha$ be implemented by translation by 1 on $\mathbf{Z}$. Note that although $M$ is finite, there is no finite normal $\alpha$-invariant trace. This is why the example does not quite belong here. Clearly $\mathbf{L}^{2}$ is $l^{2}(\mathbf{Z} \times \mathbf{Z})$ which we identify with $l^{2}(\mathbf{Z}) \otimes l(\mathbf{Z})$. Straightforward calculations show that the map $W$, 
defined by the formula $(W f)(n, m)=f(n-m, m)$, is a unitary operator on $l^{2}(\mathbf{Z} \times \mathbf{Z})$ transforming $\mathfrak{L}$ into $\{\mathbf{C} I\} \otimes \mathfrak{L}\left(l^{2}(\mathbf{Z})\right)$ and $\mathfrak{L}_{+}$into $\{\mathbf{C} I\} \otimes$ $\mathfrak{R}_{+}\left(l^{2}(\mathbf{Z})\right)$ where $\mathfrak{L}_{+}\left(l^{2}(\mathbf{Z})\right)$ is the subalgebra of all operators in $\mathfrak{L}\left(l^{2}(\mathbf{Z})\right)$ which have lower triangular matrices with respect to the usual basis for $l^{2}(\mathbf{Z})$.

EXAMPLE 2.7. Suppose that $(\Omega, m)$ is a nonatomic probability space, that $M=L^{\infty}(\Omega)$, and that $\alpha$ is implemented by an ergodic, measure preserving transformation $T$. Then $\mathfrak{L}$ is a finite factor of type II-an instance of the Murray-von Neumann group measure algebra-and $\mathfrak{L}_{+}$is closely related to algebras studied by Arveson in [3]. It turns out that his arguments show that the isomorphism class of $\mathfrak{L}_{+}$is a complete set of conjugacy invariants for $T$.

There are many more examples, evidently of marvelous complexity, and each warranting special attention. We hope to investigate these in later papers.

3. Invariant subspaces. In this section, we investigate the invariant subspace structure of the nonselfadjoint crossed products defined above. Our ultimate goal, Theorem 3.3, is to prove that when $M$ is a factor, a generalization of Beurling's Theorem is valid. In the next section, we shall prove a qualified converse of this result. Although the primary objects of study are the invariant subspaces of $\mathbf{L}^{2}$, we are able to identify the invariant subspaces of $\mathbf{L}^{p}\left(=L^{p}(\Omega, \tau)\right), 1 \leqslant p \leqslant \infty$, as well, at least when $M$ is a factor. One consequence of this is that when $M$ is a factor, every ultraweakly closed ideal in $\mathbf{H}^{\infty}$ (left, right, or two-sided) is principal and is generated by a partial isometry. This extends the analogy which exists between $H^{\infty}(\mathbf{T})$ and the polynomial ring in one variable. That is, when $M$ is a factor, $\mathbf{H}^{\infty}$ is quite properly thought of as an operator-theoretic generalization of a twisted polynomial ring.

In this paper, we use the word "subspace" to refer to a closed linear manifold in one of the spaces $L^{p}$; if the subspace is contained in $L^{\infty}$, we shall assume that it is ultraweakly closed.

Definition. Suppose that $\mathfrak{B}$ is a finite von Neumann algebra with trace $\phi$, that $\mathfrak{A}$ is finite, maximal subdiagonal algebra in $\mathfrak{B}$ with respect to $\phi$ and an appropriate expectation, and that $\mathfrak{T}$ is a subspace of $L^{p}=L^{p}(\mathfrak{B}, \phi)$. We shall say that $\Re$ is: left- or $\left(\mathfrak{L}_{+}^{-}\right)$invariant, if $\mathfrak{R}_{+} \Re \subseteq \Re$; left-reducing, if $\mathfrak{L} \subseteq \mathfrak{N}$; left-pure, if $\Re$ contains no left-reducing subspace; and left-full, if the smallest left-reducing subspace containing $\mathfrak{T}$ is all of $L^{p}$. The righthand versions of these concepts are defined similarly, and a subspace which is both left and right invariant will be called two-sided invariant.

In order to shorten the writing, whenever we refer to a subspace as being invariant, reducing, pure, or full without specifying otherwise, we intend that it is left-invariant, left-reducing, etc. We issue this caveat, however, When we refer to a two-sided invariant subspace as pure or full, we shall mean that it is 
left-pure or left-full. This does not mean that it is either right-pure or right-full, although there are circumstances, which will be identified later, when such an implication always holds.

For the remainder of this section, we will be investigating the right and left invariant subspaces of the spaces $\mathbf{L}^{p}$ and we shall restrict our attention to the nonreducing ones because, as was noted by the third author in [20], the left-reducing subspaces of $\mathbf{L}^{p}$ are of the form $R_{e} \mathbf{L}^{p}$ for a suitable projection $e$ in $\mathbf{L}^{\infty}$ while the right-reducing subspaces have the form $L_{e} \mathbf{L}^{p}$. The following proposition shows that the analysis of the invariant subspace structure of $\mathfrak{L}_{+}$ may be reduced, in part, to known results about the invariant subspaces of $L_{\delta}$. The proof is straightforward because $\mathfrak{L}_{+}$is the $\sigma$-weakly closed algebra generated by $\mathcal{L}(M)$ and $L_{\delta}$ (Theorem 2.2) and so will be omitted. ${ }^{4}$

Proposition 3.1. Let $\Re$ be an invariant subspace in $\mathrm{L}^{2}$. Then

(1) $\Re$ reduces $\mathfrak{L}(M)$;

(2) $\Re$ reduces $\mathfrak{R}$ if and only if $\Re$ reduces $L_{\delta}$;

(3) $\Re$ is pure if and only if $\bigwedge_{n \geqslant 0} L_{\delta}^{n} \mathfrak{\Re}=\{0\}$ (i.e., if and only if $L_{\delta} \mid \mathfrak{T}$ is a pure isometry); and

(4) $\mathfrak{N}$ is full if and only if $\bigvee_{n \leqslant 0} L_{\delta}^{n} \Re=\mathbf{L}^{2}$.

Before approaching our invariant subspace theorems, it is helpful and instructive to have a couple of thoughts in mind about the Beurling, Lax, Halmos theorem which describes the invariant subspaces of a bilateral shift of arbitrary multiplicity. Suppose $U$ is a unitary operator on a Hilbert space $\mathcal{H}$ and that $\mathfrak{F}$ is a subspace of $\mathcal{H}$. Then $\mathfrak{F}$ is called a wandering subspace if $U^{n} \mathfrak{F}$ and $U^{m} \mathfrak{F}$ are orthogonal when $n \neq m$. If $\mathfrak{F}$ is a wandering subspace and if $\mathcal{H}=\bigvee_{n \in \mathbf{Z}} U^{n} \mathfrak{F}=\Sigma_{n \in \mathbf{Z}} \oplus U^{n} \mathfrak{F}$, then $\mathfrak{F}$ is called complete; and if $U$ has a complete wandering subspace, then $U$ is called a bilateral shift. Observe that $L_{\delta}$ is a bilateral shift because $E_{0} \mathbf{L}^{2}$ is a complete wandering subspace. The two principal facts about bilateral shifts, for the purpose of studying their invariant subspaces, are these: (1) the dimensions of two complete wandering subspaces are the same; and (2) the dimension of any wandering subspace is dominated by that of any complete wandering subspace. To understand how the BLH theorem is a consequence of (1) and (2), suppose $\mathfrak{F}$ is some preferred complete wandering subspace for $U$ and that $\mathcal{K}_{+}$is defined to be $\Sigma_{n \geqslant 0} \oplus$ $U^{n} \mathfrak{F}$. If $\mathscr{T}$ is any pure invariant subspace for $U$, then $\mathbb{E}=\mathscr{N} \ominus U \mathscr{R}$ is a wandering subspace and $\Re=\sum_{n=0}^{\infty} U^{n} \mathbb{S}$. Since $\operatorname{dim} \mathbb{S} \leqslant \operatorname{dim} \mathfrak{F}$, there is a partial isometry $\theta$ on $\mathcal{H}$ with initial space in $\mathfrak{F}$ and final space equal to $\mathfrak{S}$. Then, by defining $\Theta=\sum_{n \in \mathbf{Z}} U^{n} \theta U^{-n}$, we obtain a partial isometry commuting with $U$ such that $\mathscr{N}=\Theta \mathcal{H}_{+}$. This, of course, is the BLH theorem.

\footnotetext{
${ }^{4}$ From now on, all results will be formulated in terms of left-invariant subspaces. We leave it to the reader to rephrase them to obtain "right-handed" statements.
} 
Assertion (1) implies that in a sense the result is independent of the complete wandering subspace chosen in advance; or, better, to put things in a completely invariant form, we may assert that the BLH theorem is tantamount to this statement: If, for $i=1,2, \Re_{i}$ is a pure invariant subspace for $U$, if $P_{i}$ is the projection onto $\mathfrak{N}_{i}$, and if $\mathfrak{F}_{i}$ is the associated wandering subspace, then $P_{1}$ and $P_{2}$ are unitarily equivalent, where the equivalence is implemented by $a$ unitary in the commutant of $U$, if and only if $\operatorname{dim} \mathfrak{F}_{1}=\operatorname{dim} \mathfrak{\mho}_{2}$.

In this paper we are interested in certain wandering subspaces for the bilateral shift $L_{\delta}$; namely, those subspaces $\mathfrak{F}$ such that the projection $p$ from $\mathbf{L}^{2}$ onto $\mathfrak{F}$ lies in the commutant of $\mathfrak{L}(M)$. But now we are no longer interested in the Hilbert space dimension of $\mathfrak{F}$, rather we must consider the relative dimension of $\mathfrak{F}$ or, more accurately, of $p$ as an element of $\mathfrak{L}(M)^{\prime}$. Theorem 3.3 confirms that when $M$ is a factor, then necessarily two complete wandering subspaces are equivalent in $\mathfrak{L}(M)^{\prime}$, every wandering subspace is dominated, in the sense of comparison of projections in $\mathcal{L}(M)^{\prime}$, by a complete wandering subspace, and, of course, a version of the BLH theorem is true. Significantly, however, when $M$ is not a factor, two complete wandering subspaces for $L_{\delta}$ (whose projections lie in $\mathfrak{L}(M)^{\prime}$ ) may not be equivalent in $\mathfrak{L}(M)^{\prime}$; indeed they may not even be comparable (see the proof of Theorem 4.1). This discovery raises the interesting and apparently very difficult problem for nonfactors of finding a minimal, comprehensive set of mutually inequivalent, complete wandering subspaces for $L_{\delta}$ such that every wandering subspace for $L_{\delta}$ whose projection belongs to $\mathfrak{L}(M)^{\prime}$ is dominated, in $\mathfrak{L}(M)^{\prime}$, by one of the subspaces in the set. To say the same thing differently, we would like to find a family $\left\{\Re_{i}\right\}_{i \in I}$ of left-full, pure, invariant subspaces such that for no two different $\mathscr{N}_{i}$ and $\mathfrak{N}_{j}$ is there a unitary $R_{v}$ in $\Re$ such that $\Re_{j}=R_{v} \Re_{i}$ and such that every pure invariant subspace $\Re$ is of the form $\Re=R_{v} \Re_{i}$ for a suitable $i$ and partial isometry $R_{v}$ in $\Re$. When expressed this way, the problem is seen to be identical (in spirit) with that encountered in the study of invariant subspaces on Riemann surfaces (cf. [1]) and in the study of invariant subspaces on compact groups with ordered duals (cf. [10]). In the first study, the indexing set $I$ is the space of certain equivalence classes of unitary representations of the fundamental group of the surface, while in the second, it is a certain cohomology group. We note that in [13], the first author has completely solved this problem for the crossed products described in Example 2.5 and has obtained significant partial results for the algebras described in Examples 2.6 and 2.7.

THEOREM 3.2. For $i=1,2$, let $\Re_{i}$ be a left-pure, invariant subspace in $\mathbf{L}^{2}$, let $q_{i}$ be the projection of $\mathbf{L}^{2}$ onto $\Re_{i}$, and let $p_{i}$ be the projection of $\mathbf{L}^{2}$ onto $\mathfrak{T}_{i} \ominus L_{\delta} \mathfrak{T}_{i}, i=1,2$. Then each $p_{i}$ lies in $\mathfrak{L}(M)^{\prime}$, and $p_{2} \preccurlyeq p_{1}$ in $\mathfrak{L}(M)^{\prime}$ if and 
only if there is a partial isometry $R_{v}$ in $\Re$ such that $q_{2}=R_{v} q_{i} R_{v}^{*}$. In this event, $\mathfrak{T}_{2}=R_{v} \Re_{1}$.

Proof. Each $q_{i}$ lies in $\mathfrak{L}(M)^{\prime}$ by Proposition 3.1 ; and since $L_{\delta}$ normalizes $\mathfrak{L}(M)$, and therefore $\mathfrak{L}(M)^{\prime}$, it follows that $p_{i}=q_{i}-L_{\delta} q_{i} L_{\delta}^{*}$ lies in $\mathfrak{L}(M)^{\prime}$ also. If $p_{2} \preccurlyeq p_{1}$ in $\mathfrak{L}(M)^{\prime}$, then there is a partial isometry $w$ in $\mathfrak{L}(M)^{\prime}$ such that $p_{2}=w w^{*}$ and $w^{*} w \leqslant p_{1}$. Since $L_{\delta}^{k} p_{i} L_{\delta}^{* k}$ is orthogonal to $L_{\delta}^{l} p_{i} L_{\delta}^{* l}$ for $l \neq k$ (i.e., the range of $p_{i}$ is a wandering subspace for $L_{\delta}$ ), it follows that the series $\sum_{n \in \mathbf{Z}} L_{\delta}^{n} w L_{\delta}^{* n}$ converges in the strong operator topology to a partial isometry $R_{v}$ which, as a calculation shows, belongs to $\mathfrak{L}^{\prime}=\Re$. Since each $\mathfrak{N}_{i}$ is pure,

$$
q_{i}=\sum_{k=0}^{\infty} L_{\delta}^{k} p_{i} L_{\delta}^{* k}
$$

and

$$
\begin{aligned}
R_{v} q_{1} R_{v}^{*} & =\left(\sum_{n=-\infty}^{\infty} L_{\delta}^{n} w L_{\delta}^{* n}\right)\left(\sum_{k=0}^{\infty} L_{\delta}^{k} p_{1} L_{\delta}^{* k}\right)\left(\sum_{m=-\infty}^{\infty} L_{\delta}^{m} w^{*} L_{\delta}^{* m}\right) \\
& =\sum_{k>0 ; m, n \in \mathbf{Z}} L_{\delta}^{n} w L_{\delta}^{* n} L_{\delta}^{k} p_{1} L_{\delta}^{* k} L_{\delta}^{m} w^{*} L_{\delta}^{* m} .
\end{aligned}
$$

Since the range of $p_{i}$ is a wandering subspace and since the initial and final spaces of $w$ are related to the $p_{i}$ as indicated above, we see that this sum collapses to

$$
\sum_{k=0}^{\infty} L_{\delta}^{k} w p_{1} w^{*} L^{* k}=\sum_{k=0}^{\infty} L_{\delta}^{k} w w^{*} L_{\delta}^{* k}=\sum_{k=0}^{\infty} L_{\delta}^{k} p_{2} L_{\delta}^{* k}=q_{2}
$$

Thus $R_{v} q_{1} R_{v}^{*}=q_{2}$ as asserted. The converse is trivial because $R_{v}$ lies in $\Re=\mathfrak{R}^{\prime} \subseteq \mathfrak{L}(M)^{\prime}$ and

$$
R_{v} p_{1} R_{v}^{*}=R_{v} q_{1} R_{v}^{*}-R_{v} L_{\delta} q_{1} L_{\delta}^{*} R_{v}^{*}=q_{2}-L_{\delta} q_{2} L_{\delta}^{*}=p_{2},
$$

showing that $p_{2} \preccurlyeq p_{1}$ in $\mathfrak{L}(M)^{\prime}$.

THEOREM 3.3. If $M$ is a factor and if $\Re$ is a left-pure, invariant subspace, then there is a partial isometry $R_{v}$ in $\Re$ such that $\mathfrak{M}=R_{v} \mathbf{H}^{2}$. Moreover, $R_{v}$ is unitary if and only if $\Re$ is full.

Proof. Let $p$ be the projection onto $\pi \ominus L_{\delta} \Re$ and note that the projection of $\mathbf{L}^{2}$ onto $\mathbf{H}^{2} \ominus L_{\delta} \mathbf{H}^{2}$ is $E_{0}$. Since $M$ is a factor, so is $\mathcal{L}(M)^{\prime}$. Therefore either $p \preccurlyeq E_{0}$, in which case the result follows from Theorem 3.2, or $E_{0} \preccurlyeq p$. In the second case there is a partial isometry $R_{v}$ in $\Re$ such that $\mathbf{H}^{2}=R_{v} \Re$. But then, since $R_{v}$ and $L_{\delta}$ commute, we find that

$$
R_{v} \mathbf{L}^{2} \supseteq R_{v}\left(\underset{n \in \mathbf{Z}}{\bigvee} L_{\delta}^{n} \Re\right)=\bigvee_{n \in \mathbf{Z}} L_{\delta}^{n} R_{v} \Re=\bigvee_{n \in \mathbf{Z}} L_{\delta}^{n} \mathbf{H}^{2}=\mathbf{L}^{2}
$$

that is, $R_{v}$ is a coisometry. Since, however, $\Re$ is finite, $R_{v}$ must be a unitary operator and we may consequently write $\mathscr{N}=R_{v}^{*} \mathbf{H}^{2}$ as asserted. The last 
statement in the theorem is now clear and the proof is complete.

The question of uniqueness in Theorem 3.3 will be answered in Proposition 3.9 after the structure of invariant subspaces in $\mathbf{L}^{p}$ is discussed. Although the results are those one expects, the proofs are sufficiently complicated to warrant inclusion here. For the remainder of this section, we will assume that $M$ is a factor.

LeMma 3.4. If $x \in \mathbf{L}^{2}$ is such that $\left[\mathbf{H}^{\infty} x\right]_{2}$ is a pure invariant subspace, then $x=a u$ where $a$ is in $\mathbf{H}^{2}$ and $u$ is a partial isometry in $\left[\mathbf{H}^{\infty} x\right]_{2}$.

Proof. By Theorem 2.3, $\left[\mathbf{H}^{\infty} x\right]_{2}=R_{u} \mathbf{H}^{2}$ for some partial isometry $R_{u}$ in $\Re$. Since $x \in\left[\mathbf{H}^{\infty} x\right]_{2}$, it follows that $x=a u, a \in \mathbf{H}^{2}$. On the other hand, since $I \in \mathbf{H}^{2}$, we see that $u$ is in $\left[\mathbf{H}^{\infty} x\right]_{2}$.

Lemma 3.5. Suppose $1 \leqslant p \leqslant 2$ and set $r=(1 / p-1 / 2)^{-1}$. If $x \in \mathbf{L}^{p}$ is such that $\left[\mathbf{H}^{\infty} x\right]_{p}$ is pure, then $x=z y$ where $y \in\left[\mathbf{H}^{\infty} x\right]_{p} \cap \mathbf{L}^{r}$ and $z \in \mathbf{H}^{2}$.

Proof. Consider the right polar decomposition of $x$ as $x=\left|x^{*}\right| v$ and observe that since $\left[\mathbf{H}^{\infty} x\right]_{p}$ is pure, so is $\left[\mathbf{H}^{\infty}\left|x^{*}\right|^{p / 2}\right]_{2}$. Indeed, if $\left[\mathbf{H}^{\infty}\left|x^{*}\right|^{p / 2}\right]_{2}$ contained $\mathrm{L}^{2} e$ for some nonzero projection $e$ in $\mathbf{L}^{\infty}$, then the final space of $e$ would be contained in the initial space of $\left|x^{*}\right|^{1-p / 2} v$ so that $\left[\mathbf{L}^{2} e\left|x^{*}\right|^{1-p / 2} v\right]_{p}$ would be a nonzero reducing subspace of $\left[\mathbf{H}^{\infty} x\right]_{p}$, contrary to assumption. Therefore, by Lemma 2.4, we may write $\left|x^{*}\right|^{p / 2}=z u$ where $z$ is in $\mathbf{H}^{2}$ and $u$ is a partial isometry in $\left[\mathbf{H}^{\infty}\left|x^{*}\right|^{p / 2}\right]_{2}$. Set $y=u\left|x^{*}\right|^{1-p / 2} v$. Then clearly $y$ is in $\mathbf{L}^{p}$ and of course $x=z y$. Finally, since $u \in\left[\mathbf{H}^{\infty}\left|x^{*}\right|^{p / 2}\right]_{2}$, we see that $y$ lies in $\left[\mathbf{H}^{\infty}\left|x^{*}\right|^{p / 2}\right]_{2}\left|x^{*}\right|^{1-p / 2} v \subseteq\left[\mathbf{H}^{\infty} x\right]_{p}$ as asserted.

THEOREM 3.6. Let $\mathfrak{N}$ be a left-pure, invariant subspace of $\mathbf{L}^{p}, 1 \leqslant p \leqslant \infty$. Then there is a partial isometry $R_{v}$ in $\Re$ such that $\Re=R_{v} H^{p}$.

Proof. We consider separately the case when $1 \leqslant p<2$ and the case when $2<p \leqslant \infty$. The second follows from the first by a duality argument. Of course the case when $p=2$ is covered by Theorem 3.3. Suppose that $1 \leqslant p<$ 2 , set $r=(1 / p-1 / 2)^{-1}$, and let $\Re=\Re \cap \mathbf{L}^{2}$. Then Corollary 1.3 shows that $\Re$ is nonzero. Clearly $\Re$ is closed in $\mathbf{L}^{2}$, left-invariant, and a moment's reflection reveals that it is pure. Consequently, by Theorem 3.3, there is a partial isometry $R_{v}$ in $\Re$ such that $\Re=R_{v} \mathbf{H}^{2}$; and from this we conclude that $R_{v} \mathbf{H}^{\mathrm{p}} \subseteq \Re$. For the reverse inclusion, choose a nonzero $x$ in $\Re$ and note that since $\mathfrak{T}$ is pure, so is $\left[\mathbf{H}^{\infty} x\right]_{p}$. Apply Lemma 3.5 to decompose $x$ as $x=z y$ with $z$ in $\mathbf{H}^{2}$ and $y$ in $\left[\mathbf{H}^{\infty} x\right]_{p} \cap \mathbf{L}^{r}$. Since $r>2$, we may conclude that

$$
\begin{aligned}
y \in\left[\mathbf{H}^{\infty} x\right]_{p} \cap \mathbf{L}^{r} \subseteq \Re \cap \mathbf{L}^{r} & =\Re \cap \mathbf{L}^{r}=\left(\mathbf{H}^{2} v\right) \cap \mathbf{L}^{r} \\
& =\left(\mathbf{H}^{2} \cap \mathbf{L}^{r}\right) v=\mathbf{H}^{r} v,
\end{aligned}
$$

where the last equality follows from Proposition 2.7 of [21]. Thus $x=z y$ lies 
in $\mathbf{H}^{2} \mathbf{H}^{r} v \subseteq R_{v} \mathbf{H}^{p}$ and $\Re=R_{v} \mathbf{H}^{p}$. Suppose now that $2<p \leqslant \infty$ and set $q=(1-1 / p)^{-1}$. Let $e_{0}$ be the right support projection of $\mathscr{N}$; i.e., $e_{0}$ is the smallest projection $e$ such that $x e=x$. We define $\Re$ to be $\left\{y \in e_{0} \mathbf{L}^{q} \mid \tau(y x)\right.$ $=0$ for all $x \in \mathfrak{R}\}$ and we assert that $\Re$ is a right-pure, right-invariant subspace. Clearly, $\mathfrak{T}$ is right-invariant. To see that it is right-pure, observe that if $e \mathbf{L}^{q} \subseteq \Re$, then $e \leqslant e_{0}$ and $0=\tau(e y x)=\tau(y x e)$ for all $y$ in $\mathbf{L}^{q}$ and all $x$ in $\Re$. Since $e \leqslant e_{0}$, it follows by definition of right support that $e=0$. By the first half of the proof, $\Re=L_{v} \mathbf{H}^{q}$ for a suitable partial isometry $L_{v}$ in $\mathfrak{R}$. By the Hahn-Banach theorem, Proposition 2.5 of [21], and the easily verified fact that $e_{0}=v v^{*}$, we may conclude that

$$
\begin{aligned}
\mathscr{N} & =\left\{x \in \mathbf{L}^{p} e_{0} \mid \tau(x y)=0, y \in \mathscr{N}\right\}=\left\{x \in \mathbf{L}^{p} e_{0} \mid \tau(x v y)=0, y \in \mathbf{H}^{q}\right\} \\
& =\left\{x \in \mathbf{L}^{p} e_{0} \mid x v \in \mathbf{H}_{0}^{p}\right\}=\mathbf{H}_{0}^{p} v^{*}=R_{v^{*}} R_{\delta} \mathbf{H}^{p} .
\end{aligned}
$$

Since $R_{v^{*}} R_{\delta}$ is a partial isomety in $\Re$, the proof is complete.

COROLlaRY 3.7. If $\mathfrak{\Im}$ is an ultraweakly closed, left ideal in $\mathbf{H}^{p}$, then there is a partial isometry $\boldsymbol{v}$ in $\mathbf{H}^{\infty}$ such that $\mathfrak{\Im}=\mathbf{H}^{\infty} v$.

Proof. It suffices to note that $\mathfrak{J}$ is an ultraweakly closed, left invariant subspace of $\mathbf{L}^{\infty}$ which is pure because it is contained in $\mathbf{H}^{\infty}$.

We shall see later that the two-sided, ultraweakly closed ideals in $\mathbf{H}^{\infty}$ are precisely those that can be written both in the form $u \mathbf{H}^{\infty}$ and in the form $\mathbf{H}^{\infty} v$ where $u$ and $v$ are unitaries in $\mathbf{H}^{\infty}$ satisfying $u^{*} \mathbf{H}^{\infty} u=\mathbf{H}^{\infty}$ and $v \mathbf{H}^{\infty} v^{*}$ $=\mathbf{H}^{\infty}$. The next corollary was promised in Remark 1.4. It follows immediately from Theorem 3.6 and the fact that $\mathbf{H}^{p}$ is the closure of $\mathbf{H}^{\infty}$ in $\mathbf{L}^{p}$.

COROllary 3.8. Every invariant subspace of $\mathbf{L}^{p}$ is the closure in $\mathbf{L}^{p}$ of the bounded elements it contains.

We conclude this section with the following proposition which establishes the degree of uniqueness in Theorems 3.3 and 3.6. It is but a minor extension of known results.

Proposition 3.9. If $R_{v_{1}}$ and $R_{v_{2}}$ are partial isometries in $\Re$, then $R_{v_{1}} \mathbf{H}^{p}=$ $R_{v_{2}} \mathbf{H}^{p}$ if and only if $R_{v_{1}}=R_{v_{2}} R_{w}$ where $R_{w}$ is a partial isometry in $\Re(M)$ whose initial space is the initial space of $R_{v_{1}}$ and whose final space is the initial space of $R_{v_{2}}$.

Proof. By the preceding results, it clearly suffices to assume $p=2$. Since $R_{v_{1}}$ and $R_{v_{2}}$ are partial isometries commuting with $L_{\delta}$-a bilateral shift-it is well known (cf. [9, p. 64]) that $R_{v_{1}} \mathbf{H}^{2}=R_{v_{2}} \mathbf{H}^{2}$ if and only if there is a partial isometry $W$ on $\mathbf{L}^{2}$, commuting with $L_{\delta}$, and satisfying these conditions: (a) $W$ and $W^{*}$ leave $\mathbf{H}^{2}$ invariant; (b) the initial space of $W$ is the final space of $R_{v_{1}}$ while the final space of $W$ is the initial space of $R_{v_{2}}$; and (c) $R_{v_{1}}=R_{v_{2}} W$. 
Conditions (b) and (c) imply that $W=R_{v_{2}}^{*} R_{v_{1}}=R_{v_{1} v_{2}^{*}}$ is a partial isometry in $\Re$, while condition (a) implies that $W$ lies in $\Re_{+} \cap \Re_{+}^{*}=\Re(M)$.

4. Maximality. Our main objective in this section is to prove the following theorem which relates the invariant subspace structure of $\mathbf{H}^{\infty}$ to the maximality (or lack of it) of $\mathbf{H}^{\infty}$ simply as a $\boldsymbol{\sigma}$-weakly closed subalgebra of $\mathbf{L}^{\infty}$. Recall that Theorem 2.2 tells us that $\mathbf{H}^{\infty}$ is maximal as a subdiagonal algebra, but as we have stated and now prove, $\mathbf{H}^{\infty}$ need not be maximal among the $\sigma$-weakly closed subalgebras of $\mathbf{H}^{\infty}$.

The fact that $H^{\infty}(\mathbf{T})$ is a maximal weak-*-closed subalgebra of $L^{\infty}(\mathbf{T})$, a fact which we shall use, is a trivial consequence of Beurling's theorem. However, close examination of the proof reveals that the principal ingredient used is the fact that all partial isometries contained in $H^{\infty}(\mathbf{T})$ are unitary or, to put the same thing differently, the nonzero, invariant subspaces contained in $H^{2}$ are all full and pure. No such assertion is true in our setting of course, and we have found the effort required to get around this considerable and surprising.

THEOREM 4.1. The following three statements are equivalent:

(1) $M$ is a factor;

(2) $\mathbf{H}^{\infty}$ is a maximal $\sigma$-weakly closed subalgebra of $\mathbf{L}^{\infty}$; and

(3) if $\mathfrak{R}$ is a two-sided invariant subspace of $\mathbf{L}^{2}$ which does not reduce $\mathfrak{L}$, then $\Re$ may be expressed as $L_{u} \mathbf{H}^{2}$ and as $R_{v} \mathbf{H}^{2}$ where $u$ and $v$ are unitary operators in $\mathbf{L}^{2}$.

Moreover, in (3), $u$ and $v$ necessarily normalize $\mathbf{H}^{\infty}$; i.e., $u^{*} \mathbf{H}^{\infty} u=\mathbf{H}^{\infty}$ and $v \mathbf{H}^{\infty} v^{*}=\mathbf{H}^{\infty}$.

We first show that (1) and (2) are equivalent and then, using (2), we prove the equivalence of (1) and (3). To prove that (1) implies (2), we require a conditioned special case.

LEMMA 4.2. If $M$ is a factor and if $\mathfrak{B}$ is a $\left\{\beta_{t}\right\}_{t \in \mathbf{R}^{-i n v a r i a n t,} \sigma \text {-weakly closed }}$ subalgebra of $\mathbf{L}^{\infty}$ containing $\mathbf{H}^{\infty}$, then either $\mathfrak{B}=\mathbf{L}^{\infty}$ or $\mathfrak{B}=\mathbf{H}^{\infty}$.

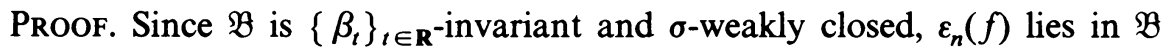
for all $f$ in $\mathfrak{B}$. So, if $\mathbf{H}^{\infty} \subsetneq \mathfrak{B}$, there is an $f$ in $\mathfrak{B}$ and an $n<0$ such that $\varepsilon_{n}(f) \neq 0$. For this $f$, we may write $\varepsilon_{n}\left(L_{f}\right)=L_{x} L_{\delta}^{n}$, for some $x \in M$. But then, since $\mathfrak{L}(M) \subseteq \mathfrak{L}_{+} \subseteq \mathfrak{L}(\mathfrak{B})$, we may write $\mathfrak{L}(M) L_{x} \mathfrak{L}(M) L_{\delta}^{n}=$ $\mathfrak{L}(M) L_{x} L_{\delta}^{n} \mathfrak{L}(M) \subseteq \mathfrak{L}(\mathfrak{B})$. Since finite factors are algebraically simple [7, p. 257], $\mathfrak{L}(M) L_{x} \mathfrak{L}(M)=\mathfrak{L}(M)$, and so $L_{\delta}^{n}$ lies in $\mathfrak{L}(\mathfrak{B})$. Finally, then, $L_{\delta}^{-1}=$ $L_{\delta}^{n} L_{\delta}^{-(n+1)}$ belongs to $\mathfrak{L}(\mathfrak{B})$ and, consequently, $\mathfrak{B}=\mathbf{L}^{\infty}$.

Proof that (1) IMPLIES (2). Suppose $M$ is a factor and that $\mathfrak{B}$ is a proper ultraweakly closed subalgebra of $\mathbf{L}^{\infty}$ containing $\mathbf{H}^{\infty}$. Form the two-sided invariant subspace $[\mathfrak{O}]_{2}$ and note that $[\mathfrak{B}]_{2} \neq \mathbf{L}^{2}$ by Corollary 1.5. Note too 
that $[\mathfrak{B}]_{2}$ does not reduce either $\mathfrak{L}_{+}$or $\mathfrak{R}_{+}$because it contains the cyclic and separating vector $I$. Finally observe that by Theorem 3.3 we need only prove that $[\mathfrak{B}]_{2}$ is left-full and left-pure. For if it is, then there is a unitary $v$ in $\mathbf{L}^{\infty}$ such that $[\mathfrak{B}]_{2}=R_{v} \mathbf{H}^{2}$. This in turn implies that

$$
\begin{aligned}
{[\mathfrak{B}]_{2} } & =R_{v}^{*} R_{v}\left[\mathfrak{L}(\mathfrak{B}) \mathbf{H}^{2}\right]_{2}=R_{v}^{*}\left[R_{v} \mathfrak{L}(\mathfrak{B}) \mathbf{H}^{2}\right]_{2} \\
& =R_{v}^{*}\left[\mathfrak{L}(\mathfrak{B}) R_{v} \mathbf{H}^{2}\right]_{2}=R_{v}^{*}\left[\mathfrak{L}(\mathfrak{B})[\mathfrak{B}]_{2}\right]=R_{v}^{*}[\mathfrak{B}]_{2}=\mathbf{H}^{2}
\end{aligned}
$$

which means that $\mathfrak{B}=\mathbf{H}^{\infty}$.

Since $\mathbf{H}^{2} \subseteq[\mathfrak{B}]_{2},[\mathfrak{B}]_{2}$ is obviously full. To show that it is pure, we begin by replacing $\mathfrak{B}$ by a potentially bigger algebra. We set $\tilde{\mathfrak{B}}=[\mathfrak{B}]_{2} \cap \mathbf{L}^{\infty}$ and we prove that $\tilde{\mathfrak{B}}$ is a proper, $\sigma$-weakly closed subalgebra of $\mathbf{L}^{\infty}$ such that $\tilde{\mathfrak{B}}[\mathfrak{B}]_{2} \subseteq[\mathfrak{B}]_{2}$. It is easy to see that $\tilde{\mathfrak{B}}$ is a subalgebra of $\mathbf{L}^{\infty}$ which is proper because $[\mathfrak{B}]_{2}$ is different from $\mathbf{L}^{2}$, and of course $\mathfrak{B} \subseteq \tilde{\mathfrak{B}}$. Since $\mathbf{L}^{2} \subseteq \mathbf{L}^{1}$, any ultraweakly convergent net in $\tilde{\mathfrak{B}}$ converges in the weak topology on $\mathbf{L}^{2}$, and since $[\mathfrak{B}]_{2}$ is weakly closed in $\mathbf{L}^{2}$, any such net has its limit in $[\mathfrak{B}]_{2}$. Thus $\tilde{\mathfrak{B}}$ is ultraweakly closed. To see that $\tilde{\mathfrak{B}}[\mathfrak{B}]_{2} \subseteq[\mathfrak{B}]_{2}$, fix $\tilde{b}$ in $\tilde{\mathfrak{B}}$ and let $\mathscr{\Re}=\{b \in$ $\left.[\mathfrak{B}]_{2} \mid \tilde{b} b \in[\mathfrak{B}]_{2}\right\}$. Since $\tilde{b}$ is in $\mathbf{L}^{\infty}$ and $[\mathfrak{B}]_{2}$ is closed in $\mathbf{L}^{2}, \mathfrak{N}$ is closed in $\mathbf{L}^{2}$. But $\mathfrak{B} \subseteq \Re$ because $[\mathfrak{B}]_{2}$ is invariant under left and right multiplication by elements in $\mathfrak{B}$. Thus $\mathfrak{N}=[\mathfrak{B}]_{2}$ and our assertions are proved.

To show that $[\mathfrak{B}]_{2}$ is pure, let $p_{\infty}$ be the projection of $\mathbf{L}^{2}$ onto $\bigcap_{n \geqslant 0} L_{\delta}^{n}[\mathfrak{B}]_{2}$. Since $[\mathfrak{B}]_{2} \neq \mathbf{L}^{2}, p_{\infty} \neq I$. Also, by Proposition 3.1, $p_{\infty}$ lies in $\mathfrak{L}^{\prime}=\mathfrak{R}$. Since, however, $[\mathfrak{B}]_{2}$ is invariant under $\Re_{+}$, so is $\bigcap_{n \geqslant 0} L_{\delta}^{n}[\mathfrak{P}]_{2}$. This implies that $p_{\infty}$ commutes with $\Re(M)$ and that $R_{\delta} p_{\infty} R_{\delta}^{*} \leqslant p_{\infty}$. Since $\mathfrak{R}$ is finite, we actually have equality: $R_{\delta} p_{\infty} R_{\delta}^{*}=p_{\infty}$. Thus, regarding $p_{\infty}$ as an element of $\mathbf{L}^{\infty}$, it belongs to the center, $3\left(\mathbf{H}^{\infty}\right)$. Finally, since $p_{\infty}=p_{\infty} \cdot I$ lies in $\bigcap_{n \geq 0} L_{\delta}^{n}[\mathfrak{B}]_{2}$ $\subseteq[\mathfrak{B}]_{2}$, we may concludde that $p_{\infty}$ lies in $3\left(\mathbf{L}^{\infty}\right) \cap \tilde{\mathfrak{B}}$. If $\mathbf{L}^{\infty}$ is a factor, then since $p_{\infty} \neq 1, p_{\infty}$ must be zero and we are done. In the contrary case, $3\left(\mathbf{L}^{\infty}\right) \cap \tilde{\mathfrak{B}}$ is a $\sigma$-weakly closed subalgebra of $3\left(\mathbf{L}^{\infty}\right)$ containing $3\left(\mathbf{L}^{\infty}\right) \cap$ $\mathbf{H}^{\infty}$. By Theorem 2.3 and the fact that $H^{\infty}(\mathrm{T})$ is a maximal weak-* closed subalgebra of $L^{\infty}(\mathbf{T})$, we find that either $3\left(\mathbf{L}^{\infty}\right) \cap \tilde{\mathfrak{B}}=3\left(\mathbf{L}^{\infty}\right) \cap \mathbf{H}^{\infty}$, in which case $p_{\infty}=0$, or $3\left(\mathbf{L}^{\infty}\right) \cap \tilde{\mathfrak{B}}=3\left(\mathbf{L}^{\infty}\right)$. But if $3\left(\mathbf{L}^{\infty}\right)$ were contained in $\tilde{\mathfrak{B}}$, then the $\sigma$-weakly closed algebra $\Subset$ generated by $\mathbf{H}^{\infty}$ and $3\left(\mathbf{L}^{\infty}\right)$ would be

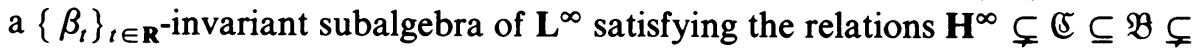
$\mathbf{L}^{\infty}$. Since this is not possible by Lemma 4.2 , we conclude once more that $p_{\infty}$ must be zero, completing the proof that (1) implies (2).

Proof that (2) Imples (1). Suppose the center of $M, 3(M)$, is nontrivial and consider two mutually exclusive and exhaustive cases.

Case 1. $\alpha$ does not act ergodically on $3(M)$.

In this case, choose an $\alpha$-invariant projection $e$ in $3(M)$ different from zero and one, and let $\mathfrak{B}$ be the $\sigma$-weakly closed algebra generated by $\mathbf{H}^{\infty}$ and $e \mathbf{L}^{\infty}$. 
Since $e$ is an $\alpha$-invariant projection in $3(M)$ it is in the center of $\mathbf{L}^{\infty}$, and it is easy to see from this that $\mathfrak{B}$ is proper and larger than $\mathbf{H}^{\infty}$. Thus in this case, $\mathbf{H}^{\infty}$ is not maximal among the $\sigma$-weakly closed subalgebras of $\mathbf{L}^{\infty}$.

Case 2. $\alpha$ acts ergocially on $3(M)$.

To prove that $\mathbf{H}^{\infty}$ is not maximal, it suffices to produce a nonreducing, left-invariant subspace $\Re$ of $\mathbf{L}^{2}$ and an element $f$ in $\mathbf{L}^{\infty} \backslash \mathbf{H}^{\infty}$ such that $L_{f} \mathfrak{N} \subseteq \mathfrak{N}$. To do this, choose a nonzero projection $e$ in $3(M)$ such that $e$ and $\alpha^{-1}(e)$ are orthogonal (we may do this by hypothesis) and set $\mathfrak{R}=\{g$ $\in \mathbf{L}^{2} \mid E_{n} g=0, n<-1$, and $\left.e E_{-1} g=E_{-1} g\right\}$. Then since $e \in Z(M)$, $\mathfrak{L}(M) \mathfrak{N} \subseteq \mathfrak{K}$ and of course $L_{\delta} \mathfrak{N} \subseteq \mathfrak{N}$; thus, $\mathfrak{L}_{+} \mathfrak{N} \subseteq \mathfrak{N}$. Also, it is clear that $L_{\delta}^{*}$ does not leave $\mathfrak{N}$ invariant, so that $\mathfrak{N}$ does not reduce $\mathfrak{L}_{+}$. However, by the condition on $e, L_{e} L_{\delta}^{*}$, which is not in $\mathfrak{L}_{+}$, does map $\Re$ into $\mathfrak{T}$. Thus, in this case too, $\mathbf{H}^{\infty}$ is not maximal among the $\sigma$-weakly closed subalgebras of $\mathbf{L}^{\infty}$, and the proof that (2) implies (1) is complete.

Proof THAT (1) IMPLIES (3). By Theorem 3.3, we need only prove that each two-sided invariant subspace which does not reduce $\mathfrak{L}_{+}$is left-full and left-pure. So let $\mathfrak{N}$ be such a space and let $p_{\infty}$ be the projection onto $\cap_{n \geq 0} L_{\delta}^{n} \Re$. Then, as in the proof that (1) implies (2), $p_{\infty}$ is different from $I$ and lies in the center of $\mathfrak{L}$. But also, $p_{\infty} \mathfrak{N} \subseteq p_{\infty} \mathbf{L}^{2}=\bigcap_{n \geqslant 0} L_{\delta}^{n} \mathfrak{R} \subseteq \Re$. Hence, by (2), $p_{\infty}$ belongs to $\mathfrak{L}_{+}$. Since $3\left(\mathbf{L}^{\infty}\right) \cap \mathbf{H}^{\infty}$ is isomorphic to $H^{\infty}(T)$ by Theorem 2.3 and $p_{\infty} \neq I$, we conslude that $p_{\infty}=0$; i.e., that $\Re$ is pure. To show that $\mathfrak{N}$ is full, let $p_{-\infty}$ be the projection onto $\bigvee_{n \in \mathbf{Z}} L_{\delta}^{n} \mathfrak{T}$. Then, as before, $p_{-\infty}$ lies in the center $\mathfrak{R}$, but this time $p_{-\infty}$ is not zero. Also, $p_{-\infty} \Re=\Re$ because $\Re \subseteq \bigvee_{n \in \mathbf{Z}} L_{\delta}^{n} \mathfrak{R}=p_{-\infty} \mathbf{L}^{2}$. Thus, by maximality once more, $p_{-\infty}$ lies in $3\left(\mathbf{L}^{\infty}\right) \in \mathbf{H}^{\infty}$ and consequently, $p_{-\infty}=1$. Thus $\Re$ is full and the proof that (1) implies (3) is complete.

Proof THAT (3) IMPLIES (2). This is actually contained in the proof that (1) implies (2). Indeed, if $\mathfrak{B}$ is a proper ultraweakly closed subalgebra of $\mathbf{L}^{\infty}$ containing $\mathbf{H}^{\infty}$, then $[\mathfrak{B}]_{2}$ is a nonreducing, two-sided invariant subspace of $\mathbf{L}^{2}$ which, by (3), must have the form $R_{v} \mathbf{H}^{2}$ for some unitary $v$ in $\mathbf{L}^{\infty}$. But then the argument in the first paragraph of the proof that (1) implies (2) shows that $\mathfrak{B}=\mathbf{H}^{\infty}$. Thus (3) implies (2).

For the last assertion of the theorem suppose that a two-sided invariant subspace has the form $L_{u} \mathbf{H}^{2}$ for a suitable unitary $u$ in $L^{\infty}$. Then since $\mathfrak{L}_{+} L_{u} \mathbf{H}^{2} \subseteq L_{u} \mathbf{H}^{2}$, we see that $L_{u}^{*} \mathfrak{L}_{+} L_{u} \mathbf{H}^{2} \subseteq \mathbf{H}^{2}$ so that $L_{u}^{*} \mathfrak{L}_{+} L_{u} \subseteq \mathfrak{L}_{+}$. If this inclusion were proper, then $\mathfrak{L}_{+}$would be properly contained in $L_{u} \mathfrak{R}_{+} L_{u}^{*}$, a proper ultraweakly closed subalgebra of $\mathfrak{R}$, contrary to (2). Thus $u^{*} \mathbf{H}^{\infty} u=$ $\mathbf{H}^{\infty}$ and a similar argument proves that $v \mathbf{H}^{\infty} v^{*}=\mathbf{H}^{\infty}$. This completes the proof of Theorem 4.1 .

There are several corollaries and modifications of Theorem 4.1 which are worth developing. The first was actually proved in the third paragraph of the proof that (1) implies (2) and so we omit the argument here. 
COROllaRy 4.3. If $e$ is a projection in $\mathbf{L}^{\infty}$ such that $L_{e} \mathbf{L}^{2}$ is invariant under $\mathfrak{L}_{+}$, then $e$ lies in $3\left(\mathbf{L}^{\infty}\right)$, so that $L_{e} \mathbf{L}^{2}$ reduces $\mathfrak{L}_{+}$.

There is a useful variation of conditon (3) in Theorem 4.1; it looks mildly weaker, but in fact it is equivalent. We present it in

Proposition 4.4. The following statement is equivalent to (3) (and hence to (1) and (2)) in Theorem 4.1.

(3') Every two-sided invariant subspace contained in $\mathbf{H}^{2}$ may be expressed in the form $L_{u} \mathbf{H}^{2}$ for a suitable unitary $u$ in $\mathbf{L}^{\infty}$.

Proof. Since $\mathbf{H}^{2}$ contains no nonzero reducing subspace, it is clear that (3) implies ( $\left.3^{\prime}\right)$. To prove the reverse implication, it suffices to prove that $\mathbf{H}^{\infty}$ is a maximal, $\sigma$-weakly closed subalgebra of $\mathbf{L}^{\infty}$. So suppose $\mathfrak{B}$ is a proper $\sigma$-weakly closed subalgebra of $\mathbf{L}^{\infty}$ containing $\mathbf{H}^{\infty}$. Then as in the proof of Theorem 4.1, $[\mathfrak{B}]_{2}$ is a proper, two-sided invariant subspace of $\mathbf{L}^{2}$ and it suffices to prove that $[\mathfrak{B}]_{2}=R_{v} \mathbf{H}^{2}$ for some unitary $v$ in $\mathbf{L}^{\infty}$. Let $\mathcal{K}=\mathbf{L}^{2} \theta$ $[\mathfrak{B}]_{2}$. Then $\mathcal{K}$ is a nonzero subspace of $\left(\mathbf{H}^{2}\right)^{\perp}=J \mathbf{H}_{0}^{2}$ and so, by Proposition $1.1, J \mathcal{K}$ is a two-sided invariant subspace of $\mathbf{H}^{2}$. By hypothesis, $J \mathscr{K}=L_{u} \mathbf{H}^{2}$ for some unitary $u$ in $\mathbf{L}^{\infty}$ and consequently,

$$
\begin{aligned}
{[\mathfrak{B}]_{2} } & =\mathbf{L}^{2} \ominus \mathscr{K}=\mathbf{L}^{2} \ominus J L_{u} \mathbf{H}^{2}=\mathbf{L}^{2} \ominus R_{u}^{*} J \mathbf{H}^{2} \\
& =\boldsymbol{R}_{u}^{*}\left(\mathbf{L}^{2} \ominus J \mathbf{H}^{2}\right)=R_{u}^{*} \mathbf{H}_{0}^{2}=R_{u}^{*} \boldsymbol{R}_{\delta} \mathbf{H}^{2} .
\end{aligned}
$$

Since $R_{u}^{*} R_{\delta}$ is a unitary in $\Re$, the proof is complete.

Theorems 3.3 and 4.1 may lead one to conjecture that a stronger form of Theorem 4.4 is true; namely, if every pure invariant subspace $\Re$ of $\mathbf{L}^{2}$ has the form $\mathfrak{R}=R_{v} \mathbf{H}^{2}$ for some partial isometry $v$ in $\mathbf{L}^{\infty}$, then $M$ is a factor. This, however, is not the case. For if $\Omega=\left\{\omega_{1}, \omega_{2}\right\}$ is a two-point space, if $M=L^{\infty}(\Omega)$, and if $\alpha$ is the trivial automorphism, then $\mathbf{L}^{\infty}$ is isomorphic to $L^{\infty}(\Omega \times \mathbf{T}), \mathbf{H}^{\infty}$ is isomorphic to $\left\{f \in L^{\infty}(\Omega \times \mathbf{T}) \mid f\left(\omega_{i}, \cdot\right) \in H^{\infty}(\mathbf{T}), i=\right.$ $1,2\}$, and it is not difficult to see that each pure invariant subspace of $\mathbf{L}^{2}$ has the desired form even though $M$ is not a factor. There are two things "wrong" with this example. The first is that $\alpha$ leaves the center of $M$ elementwise invariant. Although we have not pursued the details, it appears to be an exercise in reduction theory to prove that every pure invariant subspace of $\mathbf{L}^{2}$ has the form $R_{v} \mathbf{H}^{2}$ for some partial isometry $v$ in $\mathbf{L}^{\infty}$ if and only if $\alpha$ fixes the center of $M$ elementwise. The second thing "wrong" with the example is the fact that $3\left(\mathbf{L}^{\infty}\right) \cap \mathbf{H}^{\infty}$ is not isomorphic to $H^{\infty}(\mathbf{T})$. In view of Theorem 2.3, to say that $3\left(\mathbf{L}^{\infty}\right) \cap \mathbf{H}^{\infty}$ is isomorphic to $H^{\infty}(\mathbf{T})$ is to say that $\mathbf{L}^{\infty}$ is not a factor and $3\left(\mathbf{L}^{\infty}\right) \cap M=\{\mathbf{C} I\}$. This explains the form of the hypotheses in the following proposition which eliminates the second "defect". 
Proposition 4.5. If $3\left(\mathbf{L}^{\infty}\right) \cap M=\{\mathbf{C} I\}$ and if each invariant subspace $\Re$ of $\mathbf{H}^{2}$ has the form $\Re=R_{v} \mathbf{H}^{2}$ for some partial isometry $v$ in $\mathbf{L}^{\infty}$, then, in fact, $M$ is a factor.

Proof. We begin with an observation which is implicit in [19, Theorem 1]. Suppose $U$ is a bilateral shift on a Hilbert space $\mathscr{K}$ and that $\mathcal{K}_{+}$is a full, pure invariant subspace of $\mathcal{K}$. If $\Theta$ is a partial isometry on $\mathscr{K}$ commuting with $U$ such that $\Theta K_{+} \subseteq \mathcal{K}_{+}$, and if $P$ is the initial projection of $\Theta$, then $P K_{+} \subseteq K_{+}$. An argument for this runs as follows. Rosenblum proves that if $Q$ is the initial projection of the restriction of $\Theta$ to $\mathcal{K}_{+}, \Theta \mid \mathcal{K}_{+}$, then $Q$ commutes with the restriction $U \mid \mathscr{K}_{+}$. But $U \mid \mathscr{K}_{+}$is an isometry which has $U$ as its minimal unitary extension by hypothesis. So, by a well-known result, there is a unique projection $\tilde{Q}$ on $\mathscr{K}$ which commutes with $U$, leaves $\mathcal{K}_{+}$ invariant, and satisfies the equation $Q=\tilde{Q} \mid \mathscr{K}_{+}$. A moment's reflection reveals that $\tilde{Q}=P$.

To prove the proposition, it suffices, by Proposition 4.4, to prove that if $\pi$ is a two-sided invariant subspace of $\mathbf{H}^{2}$, then $\mathfrak{T}=R_{v} \mathbf{H}^{2}$ for some unitary $v$ in $\mathbf{L}^{\infty}$. So let $\mathscr{N}$ be such a space. By hypothesis, $\mathscr{N}=R_{v} \mathbf{H}^{2}$ for a partial isometry $v$ in $\mathbf{L}^{\infty}$. If $e=v^{*} v$, then $R_{e}=R_{v} R_{v}^{*}$, and

$$
R_{e} \mathbf{L}^{2}=R_{v} \mathbf{L}^{2}=R_{v}\left(\bigvee_{n<0} L_{\delta}^{n} \mathbf{H}^{2}\right)=\bigvee_{n<0} L_{\delta}^{n}\left(R_{v} \mathbf{H}^{2}\right)=\bigvee_{n<0} L_{\delta}^{n} \mathfrak{T}
$$

is invariant under $\Re_{+}$. By Corollary 4.4, $e$ lies in the center of $\mathbf{L}^{\infty}$. Since $\mathbf{L}^{\infty}$ is finite and $e$ is central, $v v^{*}=e$ also, i.e., $R_{e}$ is the initial projection of $R_{v}$. Since $R_{v}$ commutes with the bilateral shift $L_{\delta}$ and leaves invariant $\mathbf{H}^{2}$, a full, pure invariant subspace for $L_{\delta}$, we may apply the initial observation of the proof to conclude that $R_{e} \mathbf{H}^{2} \subseteq \mathbf{H}^{2}$. Since $e$ is Hermitian, we conclude from this that $R_{e}$ lies in $\Re_{+} \cap \Re_{+}^{*}=\Re(M)$. Thus $e$ lies in $3\left(\mathbf{L}^{\infty}\right) \cap M=\{\mathbf{C} I\}$, and since $e \neq 0, e$ is the identity operator. This proves that $v$ is unitary as promised.

The next result was promised after Corollary 3.7.

Proposition 4.6. If $M$ is a factor, then each $\sigma$-weakly closed, two-sided ideal in $\mathbf{H}^{\infty}$ can be written as $L_{u} \mathbf{H}^{\infty}$ and as $R_{v} \mathbf{H}^{\infty}$ for unitary operators $u$ and $v$ in $\mathbf{H}^{\infty}$ satisfying $u^{*} \mathbf{H}^{\infty} u=\mathbf{H}^{\infty}$ and $v \mathbf{H}^{\infty} v^{*}=\mathbf{H}^{\infty}$.

Proof. Let $\mathfrak{\Im}$ be such an ideal. By Proposition $3.7, \mathfrak{\Im}=L_{u} \mathbf{H}^{\infty}$ for a partial isometry $u$ in $\mathbf{H}^{\infty}$. On the other hand, [ $\left.\widetilde{\Im}\right]_{2}=L_{u} \mathbf{H}^{2}$ is a two-sided invariant subspace of $\mathbf{H}^{2}$ and so does not reduce $\mathfrak{L}_{+}$. By Theorem 4.1, then, $[\Im]_{2}=$ $L_{w} \mathbf{H}^{2}$ where $w$ is a unitary in $\mathbf{L}^{\infty}$ such that $w \mathbf{H}^{\infty} w^{*}=\mathbf{H}^{\infty}$. By Proposition 3.9, there is a partial isometry $L_{s}$ in $\mathfrak{L}(M)$ whose initial space is the initial space of $L_{u}$ and whose final space is the initial space of $L_{w}$ such that $L_{u}=L_{w} L_{s}$. Since $\mathfrak{L}$ is finite and $L_{w}$ is unitary, $L_{s}$ and, consequently, $L_{u}$ must be unitary; also $u \mathbf{H}^{\infty} u^{*}=w s \mathbf{H}^{\infty} s^{*} w^{*}=w \mathbf{H}^{\infty} w^{*}=\mathbf{H}^{\infty}$. This proves that $u$ has the desired 
properties, and since a similar argument applies to $v$, the proof is complete.

5. Lattices of invariant subspaces and ideals. When studying an operator algebra, it frequently is of interest to know explicitly the structure of the lattices of its invariant subspaces and ideals. In particular, it is of especial interest to know when these lattices are totally ordered. While it is clear that the lattice of $\sigma$-weakly closed left ideals, say, in $\mathbf{H}^{\infty}$ is never totally ordered, even when $M$ is a factor, the following result shows that under suitable hypotheses the lattices of two-sided invariant subspaces in $\mathbf{L}^{2}$ and of twosided $\sigma$-weakly closed ideals are totally ordered.

THEOREM 5.1. If $M$ is a factor and if $\alpha^{n}$ is outer for all $n \neq 0$, then the lattice of two-sided invariant subspaces in $\mathbf{L}^{2}$ is totally ordered, as is the lattice of $\sigma$-weakly closed, two-sided ideals in $\mathbf{H}^{\infty}$.

Proof. We attend to the invariant subspaces since the statement about ideals is proved similarly using Proposition 4.6. The hypothesis implies that $\mathbf{L}^{\infty}$ is a factor $[5$, p. 586] and so by Corollary 4.3 , no two-sided invariant subspace reduces $\mathfrak{L}_{+}$. By Theorem 4.1, each such subspace is of the form $R_{v} \mathbf{H}^{2}$ where $v$ is a unitary in $\mathbf{L}^{\infty}$ such that $v \mathbf{H}^{\infty} v^{*}=\mathbf{H}^{\infty}$. Thus, to prove the theorem, it suffices to prove that $R_{v}=R_{u} R_{\delta}^{n}$ for some integer $n$ and unitary $u$ in $M$. For this, it suffices to show that $R_{v}^{*} \Re(M) R_{v}=\Re(M)$ by Lemma 3.1 of [5]. Since $v$ normalizes $\mathbf{H}^{\infty}$, we have

$$
\begin{aligned}
R_{v}^{*} \Re(M) R_{v} & =R_{v}^{*}\left(\Re_{+} \cap \Re_{+}^{*}\right) R_{v} \\
& =\left(R_{v}^{*} \Re_{+} R_{v}\right) \cap\left(R_{v}^{*} \Re_{+}^{*} R_{v}\right)=\Re_{+} \cap \Re_{+}^{*}=\mathfrak{R}(M)
\end{aligned}
$$

as required.

Finite factors $M$ with automorphisms $\alpha$ such that $\alpha^{n}$ is outer for all $n \neq 0$ must be of type II. On the other hand, by Theorems 1 and 4 in [8], every known finite type II factor has such an automorphism. For a concrete example, assume that $M$ is hyperfinite and realized as the infinite tensor product, indexed by the integers, of two-by-two matrix algebras. In this instance one may take $\alpha$ to be that automorphism implemented by the shift transformation on the indexing set.

It strikes us that the hypotheses of Theorem 5.1, and of the corollary to follow, are too strong. We believe, but are unable to prove, that it suffices to assume that $M$ and $\mathbf{L}^{\infty}$ are factors. Of course by Theorem 2.3 these assumptions are clearly necessary.

Factors are those von Neumann algebras $\mathfrak{A}$ with the property that the von Neumann algebra generated by $\mathfrak{A}$ and $\mathfrak{U}^{\prime}$ is the full algebra of operators on the underlying Hilbert space. We now show that it is possible for $\mathfrak{L}_{+}$and $\Re_{+}$ to have a similar relationship vis-à-vis (block) triangular matrix algebras. We find this rather surprising in view of the fact that $\mathfrak{L}_{+}$seems to be unlike a 
triangular matrix algebra in so many ways.

COROLlaRY 5.2. If $M$ is a factor and if $\alpha^{n}$ is outer for all $n \neq 0$, then the weakly closed algebra $\mathfrak{A}$ generated by $\mathfrak{L}_{+}$and $\mathfrak{R}_{+}$on $\mathbf{L}^{2}$ is the algebra $\mathfrak{I}$ consisting of all operators on $\mathbf{L}^{2}$ which leave invariant each of the spaces $\sum_{k=n}^{\infty} E_{k} \mathbf{L}^{2}, n \in \mathbf{Z}$.

Proof. Since $\sum_{k=n}^{\infty} E_{k} \mathbf{L}^{2}=L_{\delta}^{n} \mathbf{H}^{2}=R_{\delta}^{n} \mathbf{H}^{2}, n \in \mathbf{Z}, \mathfrak{A}$ is certainly contained in $\mathfrak{T}$. On the other hand, by Theorem 5.1, the only subspaces left invariant by $\mathfrak{A}$ are of the form $\sum_{k=n}^{\infty} E_{k} \mathbf{L}^{2}$. To show, then that $\mathfrak{U}=\mathfrak{I}$, we may proceed in two roughly equivalent ways. The crucial step in either is to prove that $\mathfrak{A}$ contains $\left\{E_{n}\right\}_{n \in \mathbf{Z}}^{\prime}$. Once this is done, trivial calculations prove that $\left\{E_{n}\right\}_{n \in \mathbf{Z}}^{\prime}$ and $R_{\delta}$ generate $\mathfrak{T}$. If one wants to avoid these calculations and follow an alternate route, one need only note that $\left\{E_{n}\right\}_{n \in \mathbf{Z}}^{\prime}$ contains a m.a.s.a. in the full algebra of operators on $\mathbf{L}^{2}$. Since the lattice of subspaces invariant under $\mathfrak{A}$ is totally ordered, this and Theorem 9.24 in [17] imply that $\mathfrak{A}=\mathfrak{T}$.

We show that $\left\{E_{n}\right\}_{n \in \mathbf{Z}}^{\prime}=\{\mathfrak{L}(M), \mathfrak{R}(M)\}^{\prime \prime}$ which in turn is contained in $\mathfrak{A}$. For this, we denote the operators in the left regular representation of $M$ on $L^{2}(M, \phi)$ by $l_{x}, x \in M$, and those in the right regular representation by $r_{x}$. On $\mathbf{L}^{2}$, which we identify with a direct sum of copies of $L^{2}(M, \phi)$, the operators $L_{x}$ and $R_{x}, x \in M$, have these matricial representations:

and
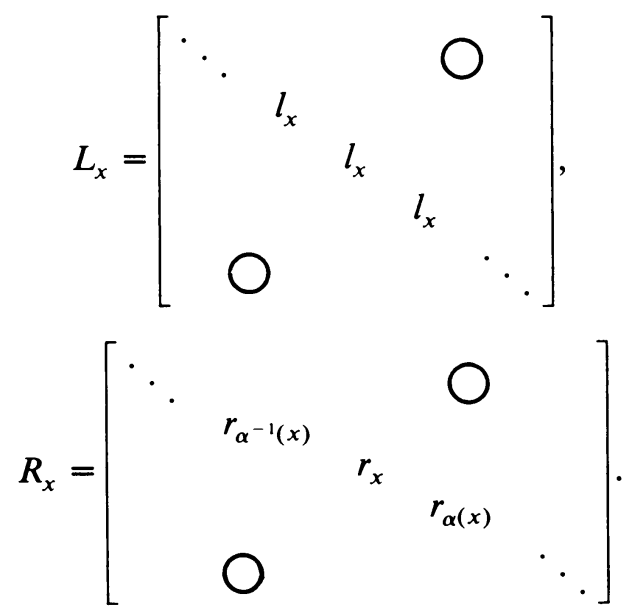

Any operator $A$ in $\{\mathfrak{L}(\boldsymbol{M}), \mathfrak{R}(\boldsymbol{M})\}^{\prime}$ is in $\mathfrak{L}(\boldsymbol{M})^{\prime}$ and so has a matricial representation $A=\left[r_{x_{n, m}}\right]$ for suitable $x_{n, m}$ in $M$. In order for $A$ to commute with $\Re(M)$, it is necessary and sufficient that for each pair $(n, m)$, the equation $\alpha^{n}(y) x_{n, m}=x_{n, m} \alpha^{m}(y)$ hold for all $y$ in $M$. This, in turn, is equivalent to the validity of the equation

$$
y \alpha^{-n}\left(x_{n, m}\right)=\alpha^{-n}\left(x_{n, m}\right) \alpha^{m-n}(y)
$$

for all $y$ in $M$. If $n=m$, then equation (1) shows that $x_{n, n}$ lies in $3(M)$. Since 
$M$ is a factor, each $x_{n, n}$ is a scalar (which may depend on $n$ ). If, on the other hand, $n \neq m$ and if $x_{n, m} \neq 0$, then a straightforward argument using the polar decomposition of $\alpha^{-n}\left(x_{n, m}\right)$ and equation (1) shows that $\alpha^{m-n}$ is inner, contrary to hypothesis. (The details are on p. 203 of [25].) Thus we find that $\{\mathfrak{L}(\boldsymbol{M}), \mathfrak{R}(M)\}^{\prime} \subseteq\left\{E_{n}\right\}_{n \in \mathbf{Z}}^{\prime \prime}$ so that $\left\{E_{n}\right\}_{n \in \mathbf{Z}}^{\prime} \subseteq\{\mathfrak{L}(M), \mathfrak{R}(M)\}^{\prime \prime}$. Since the reverse inclusion is clear, the proof is complete.

6. Which subdiagonal algebras are crossed products? We have seen that the validity of the BLH theorem in crossed products has a number of surprising and strong consequences. One might well ask, therefore, what the most general setting is in which this theorem is valid. We believe that the following result is the best one can hope for in a search for ultimate generality.

We fix once and for all a finite von Neumann algebra $\mathfrak{B}$ with trace $\phi$ and a subalgebra $\mathfrak{A}$ of $\mathfrak{B}$ which is finite, maximal, subdiagonal algebra in $\mathfrak{B}$ with respect to $\phi$ and expectation $\Phi$ mapping $\mathscr{B}$ onto $\mathfrak{D}=\mathfrak{A} \cap \mathfrak{U}^{*}$. We adopt the notation of $\S 1$ and write $L^{2}=L^{2}(\mathfrak{B}, \phi), H^{2}=[\mathfrak{A}]_{2}$, etc.

THEOREM 6.1. If every nonzero subspace $\Re$ of $H^{2}$ which is invariant under $\mathfrak{L}_{+}$and $\Re_{+}$has the form $\mathfrak{R}=R_{v} H^{2}$ for some unitary $v$, then there is a normal *-automorphism $\alpha$ of $\mathfrak{D}$ preserving $\phi$ such that $\mathfrak{B}$ is isomorphic to the crossed product $\mathbf{L}^{\infty}$ determined by $\mathfrak{D}$ and $\alpha$ in such a way that $\mathfrak{A}$ becomes identified with the corresponding space $\mathbf{H}^{\infty}$.

This and Proposition 4.4 immediately yield

COROLlaRY 6.2. $\mathfrak{D}$ is a factor.

We break the proof of Theorem 6.1 up into a series of lemmas. The first thing to observe is that $H_{0}^{2}$, which is the closure in $L^{2}$ of the kernel of $\Phi$ restricted to $\mathfrak{A}$, is a two-sided invariant subspace of $H^{2}$. By hypothesis, there is a unitary $v$ in $\mathfrak{B}$ such that $R_{v} H^{2}=H_{0}^{2}$. What is required is a careful analysis of $v$.

LeMmA 6.3. If $v$ is a unitary in $B$ such that $R_{v} H^{2}=H_{0}^{2}$, then for all $n$ in $\mathbf{Z}$, $R_{v}^{n} H^{2}=L_{v}^{n} H^{2}$.

Proof. If $H_{0}^{2}=R_{v} H^{2}$, then by Proposition 1.1, $H^{2}=L^{2} \ominus J H_{0}^{2}=L^{2} \ominus J R_{v} H^{2}=L^{2} \ominus L_{v}^{*} J H^{2}=L_{v}^{*}\left(L^{2} \ominus J H^{2}\right)=L_{v}^{*} H_{0}^{2}$, so that $R_{v} H^{2}=H_{0}^{2}=L_{v} H^{2}$. If $n \geqslant 1$ and if $R_{v}^{n} H^{2}=L_{v}^{n} H^{2}$, then $R_{v}^{n+1} H^{2}=R_{v}\left(R_{v}^{n} H^{2}\right)=R_{v}\left(L_{v}^{n} H^{2}\right)=L_{v}^{n}\left(R_{v} H^{2}\right)=L_{v}^{n}\left(L_{v} H^{2}\right)=L_{v}^{n+1} H^{2}$. Thus $L_{v}^{n} H^{2}=R_{v}^{n} H^{2}$ for all $n \geqslant 0$. But if $n<0$, then using Proposition 1.1 once more and the fact that

$$
R_{v}^{* n} H_{0}^{2}=R_{v}^{*(n-1)} H^{2}=L_{v}^{*(n-1)} H^{2}=L_{v}^{* n} H_{0}^{2},
$$


we find that

$$
\begin{aligned}
R_{v}^{n} H^{2} & =J^{2} R_{v}^{n} H^{2}=J\left(L_{v}^{* n}\left(J H^{2}\right)\right)=J\left(L_{v}^{* n}\left(L^{2} \ominus H_{0}^{2}\right)\right) \\
& =J\left(L^{2} \ominus L_{v}^{* n} H_{0}^{2}\right)=J\left(L^{2} \ominus R_{v}^{* n} H_{0}^{2}\right)=J\left(R_{v}^{* n}\left(J H^{2}\right)\right)=L_{v}^{n} H^{2} .
\end{aligned}
$$

LEMMA 6.4. $\mathfrak{U}$ is a maximal, $\sigma$-weakly closed subalgebra of $\mathfrak{B}$.

Proof. Using Corollary 1.5 , the proof requires only a notational change in the proof of Proposition 4.4. Let $₫$ be a proper $\sigma$-weakly closed subalgebra of $\mathfrak{B}$ containing $\mathfrak{X}$. Then $\mathscr{K}=L^{2} \ominus[\mathfrak{E}]_{2}$ is nonzero by Corollary 1.5 and $J \mathscr{K}$ is a two-sided invariant subspace in $H^{2}$. By hypothesis there is a unitary $v$ in $\mathfrak{B}$ such that $J \mathscr{K}=R_{v} H^{2}$. It follows that [巨్ $]_{2}=L_{v}^{*} H_{0}^{2}$. Since $H_{0}^{2}$ is itself of the form $L_{w} H^{2}$ for a suitable unitary $w$, by hypothesis and Lemma 6.3, we conclude that $[\mathbb{E}]_{2}=L_{u} H^{2}$ where $u=v^{*} w$ is unitary. As in the proof that (1) implies (2) in Theorem 4.1, it follows that $\mathbb{E}=\mathfrak{U}$.

LEMMA 6.5. Let $v$ be a unitary in $\mathfrak{B}$ such that $H_{0}^{2}=R_{v} H^{2}$. Then $v \mathfrak{D} v^{*}=\mathfrak{D}$.

Proof. By Lemma 6.3, $H_{0}^{2}=L_{v} H^{2}$ and since $\mathfrak{L}(\mathfrak{D}) \subseteq \mathfrak{R}_{+}$, we find that

$$
L_{v}^{*} \mathfrak{Q}(\mathfrak{D}) L_{v} H^{2}=L_{v}^{*} \mathfrak{L}(\mathfrak{D}) H_{0}^{2} \subseteq L_{v}^{*} H_{0}^{2}=H^{2} .
$$

Thus $L_{v}^{*} \mathfrak{L}(\mathfrak{D}) L_{v} \subseteq \mathfrak{R}_{+}$. Since $\mathfrak{L}(\mathfrak{D})$ is selfadjoint, $L_{v}^{*} \mathfrak{R}(\mathfrak{D}) L_{v} \subseteq \mathfrak{L}_{+} \cap \mathfrak{L}_{+}^{*}=$ $\mathfrak{L}(\mathfrak{D})$. On the other hand,

$$
L_{v} \mathfrak{R}(\mathfrak{D}) L_{v}^{*} H_{0}^{2}=L_{v} \mathfrak{R}(\mathfrak{D}) H^{2} \subseteq L_{v} H^{2}=H_{0}^{2} .
$$

But $H_{0}^{2}$ is also invariant under $\mathfrak{L}_{+}$and $\mathfrak{A}$ is a maximal $\sigma$-weakly closed subalgebra of $\mathfrak{B}$ by Lemma 6.4; consequently $L_{v} \mathfrak{R}(\mathfrak{D}) L_{v}^{*} \subseteq \mathfrak{L}_{+} \cap \mathfrak{L}_{+}^{*}=\mathfrak{L}(\mathfrak{D})$. Thus $L_{v} \mathfrak{L}(\mathfrak{D}) L_{v}^{*}=\mathfrak{L}(\mathfrak{D})$; or equivalently, $v \mathfrak{D} v^{*}=\mathfrak{D}$ as was to be proved.

PROOF OF THEOREM 6.1. Fix once and for all a unitary operator $v$ in $\mathfrak{B}$ such that $R_{v} H^{2}=H_{0}^{2}=L_{v} H^{2}$ and consider the selfadjoint subalgebra $\tilde{\mathfrak{B}}$ of $\mathfrak{B}$ generated by $\mathfrak{D}$ and $v$. (We are not closing $\tilde{\mathfrak{B}}$ in any topology; it consists merely of finite sums of finite products with terms in $\mathfrak{D} \cup\left\{v^{n}\right\}_{n \in \mathbf{z}}$.) It is clear that when $\mathfrak{B}$ is viewed as a Hilbert algebra, $\tilde{\mathfrak{B}}$ is a sub-Hilbert algebra. Let $\alpha$ be the automorphism of $\mathfrak{D}$ implemented by $v$; i.e., $\alpha(d)=v d v^{*}, d \in \mathfrak{D}$. Note that $\alpha$ preserves $\phi$ and let $\mathbf{L}_{0}^{2}$ be the Hilbert algebra associated with $\mathfrak{D}$, $\alpha$, and $\phi$ as in $\S 2$. For $x=\sum_{k \in \mathbf{Z}} d_{k} v^{k}$ in $\tilde{\mathfrak{B}}$, we define $w x=f$ in $\mathbf{L}_{0}^{2}$, where $f(k)=d_{k}$, and we prove that $W$ is well defined and isometric at the same time. First note that since $L_{v}^{n}[\mathfrak{D}]_{2} \subseteq H_{0}^{2} \subseteq[\mathfrak{D}]_{2}^{\perp}$ for all positive integers $n$, $[\mathfrak{D}]_{2}$ is a wandering subspace for the unitary operator $L_{v}$. Now the Hilbert algebra norm of $x=\sum_{k \in \mathbf{Z}} d_{k} v^{k}$ in $\tilde{\mathfrak{B}}$ is given by the formula $\|x\|^{2}=\phi\left(x^{*} x\right)$ which is the same as

$$
\sum_{k} \sum_{l} \phi\left(v^{* l} d_{l}^{*} d_{k} v^{k}\right)=\sum_{k} \sum_{l} \phi\left(v^{k-l} d_{l}^{*} d_{k}\right)=\sum_{k} \phi\left(d_{k}^{*} d_{k}\right)=\sum_{k}\left\|d_{k}\right\|^{2},
$$

the Hilbert algebra norm of $W x$ in $\mathbf{L}_{0}^{2}$. Thus $W$ is well defined and isometric 
and it is a simple matter to check that $W$ is a Hilbert algebra isomorphism from $\tilde{\mathfrak{B}}$ onto $\mathbf{L}_{0}^{2}$. To complete the proof, then, we need only show that $[\tilde{\mathfrak{B}}]_{2}=L^{2}$ and that

$$
\sum_{n=0}^{\infty} L_{v}^{n}[\mathfrak{D}]_{2}=\sum_{n=0}^{\infty}\left[v^{n} \mathfrak{D}\right]_{2}=H^{2}
$$

(cf. [7, Proposition 1 on p. 72]). This is tantamount to showing that $[\mathfrak{D}]_{2}$ is a complete wandering subspace for $L_{v}$. Consider first the space $\pi=H^{2} \ominus$ $\sum_{n=0}^{\infty} L_{v}^{n}[\mathfrak{D}]_{2}$. Since $[\mathfrak{D}]_{2}=H^{2} \ominus H_{0}^{2}=H^{2} \ominus L_{v} H^{2}$, it is well known and easy to show that $\mathscr{N}=\bigcap_{n \geqslant 0} L_{v}^{n} H^{2}$. Thus $\Re$ is right-invariant. On the other hand, Lemma 6.3 shows that $\mathfrak{T}=\bigcap_{n \geqslant 0} R_{v}^{n} H^{2}$ so that $\mathfrak{T}$ is left-invariant as well. Since $\Re$ is contained in $H^{2}$, $\mathscr{N}$ does not reduce $\mathfrak{L}_{+}$unless $\mathscr{R}=\{0\}$. But if $\mathscr{R} \neq\{0\}$, then by Lemma 6.4, $\mathfrak{L}_{+}=\left\{L_{x} \in \mathfrak{L} \mid L_{x} \mathfrak{R} \subseteq\right.$ $\mathfrak{R}\}$. Since, however, $L_{v}^{*} \mathfrak{N}=\mathfrak{N}$, it follows that if $\mathfrak{N} \neq\{0\}$, then $L_{v}^{*}$ lies in $\mathfrak{L}_{+}$. This and the fact that $L_{v}$ is in $\mathfrak{L}_{+}$would imply that $v$ is in $\mathfrak{D}$, which it clearly is not since $[\mathfrak{D}]_{2}$ is a wandering subspace for $L_{v}$. Thus $\Re=0$ and $H^{2}=\sum_{n=0}^{\infty} L_{v}^{n}[\mathfrak{D}]_{2}$. To finish the proof, note that

$$
H_{0}^{2}=L_{v} H^{2}=\sum_{n=0}^{\infty} L_{v}^{n+1}[\mathfrak{D}]_{2}=\sum_{n=1}^{\infty} L_{v}^{n}[\mathfrak{D}]_{2},
$$

and so

$$
J H_{0}^{2}=\sum_{n=1}^{\infty} R_{v}^{* n}[J \mathfrak{D}]_{2}=\sum_{n=-\infty}^{-1} R_{v}^{n}[\mathfrak{D}]_{2}=\sum_{n=-\infty}^{-1} L_{v}^{n}[\mathfrak{D}]_{2},
$$

where the last equality is justified by Lemma 6.5. Thus, by Proposition 1.1,

$$
\begin{aligned}
L^{2} & =H^{2} \oplus J H_{0}^{2}=\sum_{n=0}^{\infty} L_{v}^{n}[\mathfrak{D}]_{2} \oplus \sum_{n=-\infty}^{-1} L_{v}^{n}[\mathfrak{D}]_{2} \\
& =\sum_{n=-\infty}^{\infty} L_{v}^{n}[\mathfrak{D}]_{2},
\end{aligned}
$$

and the proof is complete.

ADDED IN PROOF. The conjecture made in Remark 1.4 is correct and is proved in the forthcoming paper by the third author, entitled $A$ note on invariant subspaces for finite maximal subdiagonal algebras, which will appear in the Proceedings of the American Mathematical Society.

\section{REFERENCES}

1. M. B. Abrahamse and R. G. Douglas, Subnormal operators related to multiply connected domains, Advances in Math. 19 (1976), 106-148.

2. W. B. Arveson, Analyticity in operator algebras, Amer. J. Math. 89 (1967), 578-642.

3. Operator algebras and measure preserving automorphisms, Acta Math. 118 (1967), 95-109.

4. 217-243. On groups of automorphisms of operator algebras, J. Functional Analysis 15 (1974), 
5. H. Behncke, Automorphisms of crossed products, Tôhoku Math. J. 21 (1969), 580-600.

6. J. Dixmier, Formes linéaires sur un anneau d'opérateurs, Bull. Soc. Math. France 81 (1953), 9-39.

7. Les algèbres d'opérateurs dans l'espace Hilbertien, Gauthier-Villars, Paris, 1969.

8. J. Feldman and C. Moore, Ergodic equivalence relations, cohomology, and von Neumann algebras. II, Trans. Amer. Math. Soc. 234 (1977), 325-359.

9. H. Helson, Lectures on invariant subspaces, Academic Press, London and New York, 1964.

10. New York, 1975. Analyticity on compact abelian groups, in Algebras in Analysis, Academic Press,

11. R. I. Loebl and P. S. Muhly, Analyticity and flows in von Neumann algebras, J. Functional Analysis 29 (1978), 214-252.

12. G. Mackey, Point realizations of transformation groups, Illinois J. Math. 6 (1962), 327-335.

13. M. McAsey, Invariant subspaces of nonselfadjoint crossed products, Thesis, Univ. of Iowa, May, 1978.

14. F. J. Murray and J. von Neumann, On rings of operators, Ann. of Math. (2) 37 (1936), 116-229.

15. E. Nelson, Notes on noncommutative integration, J. Functional Analysis 15 (1974), 103-116.

16. D. Olesen, On spectral subspaces and their applications to automorphism groups, Physics Theory Seminar 1973-1974, U.E.R. Scientifique de Luminy (Université d'Aix-Marseille II), 1974.

17. H. Radjavi and P. Rosenthal, Invariant subspaces, Springer-Verlag, New York, Heidelberg, and Berlin, 1973.

18. V. A. Rohlin, Selected topics from the metric theory of dynamical systems, Amer. Math. Soc. Transl. (2) 49 (1966), 171-240.

19. M. Rosenblum, Vectorial Toeplitz operators and the Fejer-Riesz theorem, J. Math. Anal. Appl. 23 (1968), 139-147.

20. K.-S. Saito, The Hardy spaces associated with a periodic flow on a von Neumann algebra, Tôhoku Math. J. 29 (1977), 69-75.

21. On non-commutative Hardy spaces associated with flows on finite von Neumann algebras, Tôhoku Math. J. 29 (1977), 585-595.

22. I. E. Segal, A non-commutative extension of abstract integration, Ann. of Math. (2) 57 (1953), $401-457$.

23. T. Srinivasan and J.-K. Wang, Weak-* Dirichlet algebras, Proc. Internat. Sympos. on Function Algebras (Tulane Univ., 1965), Scott-Foresman, Chicago, Ill., 1966, pp. 216-249.

24. M. Takesaki, Duality for crossed products and the structure of von Neumann algebras of type III, Acta Math. 131 (1973), 249-310.

25. G. Zeller-Meier, Produits croisés d'une $C^{*}$-algèbre par un groupe d'automorphismes, J. Math. Pures Appl. (9) 47 (1968), 101-239.

Department of Mathematics, University of Iowa, Iowa City, Iowa 52242 (Current address of P. S. Muhly)

Department of Mathematics, Nitgata University, Ikarashi 2-8050, Ningata 950-21 Japan (Current address of K.-S. Saito)

Current address (Michael McAsey): Department of Mathematics, Western Michigan University, Kalamazoo, Michigan 49008 